\title{
Neuromedin B Promotes Chondrocyte Differentiation of Mesenchymal Stromal Cells Via Calcineurin and Calcium Signaling
}

\author{
Marie Maumus \\ INSERM U1183 \\ Guillaume Fonteneau \\ Inserm U1183 \\ Maxime Ruiz \\ INSERM U1183 \\ Said Assou \\ INSERM U1183 \\ Hassan Boukhaddaoui \\ INSERM \\ Philippe Pastoureau \\ Les Laboratoires Servier SASGidy \\ Frédéric De Ceuninck \\ servier \\ Christian Jorgensen \\ INSERM U1183 \\ Danièle Noël ( $\nabla$ daniele.noel@inserm.fr) \\ INSERM https://orcid.org/0000-0002-3498-3776
}

\section{Research}

Keywords: mesenchymal stem cell, chondrogenesis, cartilage, transcriptomic, NMB

Posted Date: November 11th, 2020

DOl: https://doi.org/10.21203/rs.3.rs-101233/v1

License: (c) (i) This work is licensed under a Creative Commons Attribution 4.0 International License.

Read Full License

Version of Record: A version of this preprint was published at Cell \& Bioscience on October 18th, 2021. See the published version at https://doi.org/10.1186/s13578-021-00695-1. 


\section{Abstract}

Background: Articular cartilage is a complex tissue with poor healing capacities. Current approaches for cartilage repair based on mesenchymal stromal cells (MSCs) are often disappointing because of the lack of relevant differentiation factors that could drive MSC differentiation towards a stable mature chondrocyte phenotype.

Methods: We used a large-scale transcriptomic approach to identify genes that are modulated at early stages of chondrogenic differentiation using the reference cartilage micropellet model.

Results: We identified several modulated genes and selected neuromedin B (NMB) as one of the early and transiently modulated genes. We found that the timely regulated increase of NMB was specific for chondrogenesis and not observed during osteogenesis or adipogenesis. Furthermore, NMB expression levels correlated with the differentiation capacity of MSCs and its inhibition resulted in impaired chondrogenic differentiation indicating that NMB is required for chondrogenesis. We further showed that $\mathrm{NMB}$ activated the calcineurin activity through a $\mathrm{Ca}^{++}$-dependent signaling pathway.

Conclusion: NMB is a newly described chondroinductive bioactive factor that upregulates the key chondrogenic transcription factor Sox9 through the modulation of $\mathrm{Ca}^{2+}$ signaling pathway and calcineurin activity.

\section{Background}

Articular cartilage is located at the extremities of long bones and is essential for frictionless joint movement. Chondrocytes are the only mature cells found in this tissue, whose function is to secrete the extracellular matrix (ECM). The ECM is mainly composed of proteoglycans and collagens, which confer to cartilage its viscoelastic and mechanical properties. However, articular cartilage has little capacity for self-repair and cannot regenerate after severe joint injuries or advanced osteoarthritis. Common surgical treatments use chondroplasty, microfracture and mosaicplasty while more modern cartilage repair strategies include matrix-assisted chondrocyte implantation or autologous matrix-induced chondrogenesis [1]. Cartilage engineering approaches based on mesenchymal stromal/stem cells (MSCs) have provided promising results in pre-clinical models when MSCs are used in combination with scaffolds and differentiation factors such as transforming growth factor $\beta$ (TGF $\beta$ ) [2]. Yet, cartilage repair procedures may be disappointing on the longer term and lead to a non-functional fibrous or hypertrophic cartilage tissue. These results might be explained by the inclusion of patients with lower probability of successful repair (large lesions, older age, inflammation, comorbidity...) and/or the lack of a relevant differentiation factor that could be used to drive MSC differentiation towards a stable mature chondrocyte phenotype.

Chondrogenesis is a complex process in which, MSCs differentiate into chondrocytes under the spatiotemporal control of different growth and transcription factors or microRNAs [3]. However, a complete understanding of the molecular mechanisms controlling differentiation is still missing. Several 
transcriptomic analyses have been performed on MSCs, embryonic stem cells or induced pluripotent stem cells that were differentiated towards chondrocytes. Many microRNAs, long non-coding RNAs and mRNA transcripts have been found to be modulated during the differentiation process [4-14]. In addition, some gene transcripts have been reported to be predictive markers of high differentiation potential. As examples, miR-210 and miR-630 were shown to be positive regulators of chondrogenesis, and the expression levels of cyclin-dependent kinase 4 inhibitor 2D (CDKN2D), CD74, and transglutaminase 2 (TGM2) to be 10-fold higher in highly potent MSCs $[15,16]$. In the different studies, the primary model used was the micropellet culture model, obtained after aggregation and culture of stem cells in a chondrogenic medium containing TGF $\beta 3$ during 3 weeks [17]. Gene expression was compared between chondrocyte samples (TGF 33 -induced micropellets, different time points) and MSCs (day 0, before aggregation) but never with MSCs cultured in micropellets (3D culture conditions in absence of chondroinductive factors).

The aim of the present study was to identify new genes involved in the early steps of chondrogenesis by large-scale transcriptomic analysis. This approach should identify genes governing the initiation of chondrocyte differentiation, and genes predicting MSCs with high chondrogenic potential. The originality of our approach was to exclude the genes modulated by the 3D culture conditions, thereby highlighting the genes modulated during the chondrogenic differentiation of MSCs.

\section{Methods}

\section{Cell isolation and culture}

Human bone marrow (BM)-MSCs were characterized by their immunophenotype $\mathrm{CD} 11 \mathrm{~b} / \mathrm{CD} 19 \% \mathrm{CD} 34 / \mathrm{CD} 45 / \mathrm{CD} 73^{+} / \mathrm{CD}{ }^{+} / \mathrm{CD} 105^{+}$and trilineage differentiation potential as previously described and used before passage [18]. They were maintained in proliferative medium consisting in aMEM (Lonza; Levallois-Perret, France), $1 \mathrm{ng} / \mathrm{mL}$ of basic fibroblast growth factor (bFGF; R\&D Systems; Noyal Châtillon sur Seiche, France), $100 \mu \mathrm{g} / \mathrm{mL}$ penicillin/streptomycin (PS; Lonza), 2 mM glutamine (Lonza) and supplemented with $10 \%$ fetal calf serum (FCS). Cartilage slices $\left(<1 \mathrm{~mm}^{3}\right)$ were recovered from the knee joints of osteoarthritic (OA) patients and incubated in $2.5 \mathrm{mg} / \mathrm{mL}$ pronase (Sigma-Aldrich, Saint-Quentin-Fallavier, France) at $37^{\circ} \mathrm{C}$ for $1 \mathrm{~h}$ followed by an incubation with $2 \mathrm{mg} / \mathrm{mL}$ type II collagenase (Sigma) at $37^{\circ} \mathrm{C}$ overnight. Digested cartilage tissues were filtrated through a $70 \mu \mathrm{m}$ cell strainer and chondrocytes were seeded at 25,000 cells $/ \mathrm{cm}^{2}$ in DMEM high glucose (Lonza) supplemented with $2 \mathrm{mmol} / \mathrm{mL}$ glutamine, $100 \mathrm{U} / \mathrm{mL}$ PS, $1.25 \mu \mathrm{g} / \mathrm{mL}$ Amphotericin B, $5 \mu \mathrm{g} / \mathrm{mL}$ insulin, $5 \mathrm{ng} / \mathrm{mL}$ bFGF and $10 \%$ FCS until the end of passage 0 .

For proliferation assays, BM-MSCs were plated at 10,000 cells/well in 6-well plates in proliferative medium. After 5 days of culture, viable cells were counted after Trypan blue exclusion using a Malassez hemocytometer.

\section{Differentiation assays}


Chondrogenic differentiation of BM-MSCs was induced by culture in micropellets for 21 days. Briefly, 250,000 cells were pelleted by centrifugation in $15 \mathrm{~mL}$ conical tubes (6-8 tubes) and cultured in 3D conditions consisting in DMEM supplemented with $100 \mu \mathrm{g} / \mathrm{mL}$ PS, $0.35 \mathrm{mM}$ proline, $0.1 \mu \mathrm{M}$ dexamethasone, $0.17 \mathrm{mM}$ ascorbic acid-2-phosphate, $1 \mathrm{mM}$ pyruvate sodium, 1\% insulin-transferrinselenic acid (Lonza). In chondrogenic conditions, TGFß3 (10 ng/mL; R\&D Systems) was added at each medium change. For RNA extraction, micropellets were lyzed in RLT buffer according to the recommendations of the supplier (Qiagen; Les Ulis, France) at different time points (day 0.5, 1, 2, 3, 7, 14 and 21) and stored at $-80^{\circ} \mathrm{C}$.

For osteoblastogenesis, BM-MSCs were plated at 3,000 cells/ $\mathrm{cm}^{2}$ and cultured in osteogenic medium (DMEM containing 10\% FCS, $10 \mathrm{mM} \beta$-glycerophosphate, $0.1 \mu \mathrm{M}$ dexamethasone, $70 \mu \mathrm{M}$ ascorbic acid-2phosphate and $100 \mu \mathrm{g} / \mathrm{mL}$ PS). Cells were either lyzed in RLT buffer (Qiagen) at different time points (day $0.5,1,2,3,7,14$ and 21 ) and stored at $-80^{\circ} \mathrm{C}$ until RNA extraction or fixed with $95 \%$ ethanol for Alizarin Red S staining at day 21.

For adipogenic differentiation, BM-MSCs were plated at 9,000 cells $/ \mathrm{cm}^{2}$ and cultured in proliferative medium for 5 days. The differentiation medium (DMEM-F12 containing 5\% FCS, $100 \mu \mathrm{g} / \mathrm{mL}$ PS, $16 \mu \mathrm{M}$ biotin, $18 \mu \mathrm{M}$ panthotenic acid, $100 \mu \mathrm{M}$ ascorbic acid, $60 \mu \mathrm{M}$ indomethacin, $450 \mu \mathrm{M}$ isobutylmethylxanthine, $1 \mu \mathrm{M}$ dexamethasone, $1 \mu \mathrm{M}$ rosiglitazone) was then added and changed every 3 days until day 21. Cells were either lyzed in RLT buffer at different time points (day 0.5, 1, 2, 3, 7, 14 and 21) and stored at $-80^{\circ} \mathrm{C}$ until RNA extraction or fixed with $2.5 \%$ glutaraldehyde for Oil Red 0 staining at day 21.

\section{RNA isolation and microarray hybridation}

Total RNA was extracted using the RNeasy mini kit (Qiagen) following the supplier's recommendations. RNA concentration was measured using a Nanodrop (ThermoFisher Scientific; Illkirch, France) and their integrity by using the Agilent 2100 Bioanalyzer (Agilent Technologies). Total RNA (150-200 ng) was reverse-transcribed after ribosomal fraction depletion from $2 \mu \mathrm{g}$ total RNA, then hybridized onto GeneChip Human Exon 1.0 ST Affymetrix microarray. Ambion WT Expression Kits were used for amplification and Affymetrix WT Terminal Labeling Kits were used for labeling.

\section{Data processing and gene expression profile analysis}

After image processing with the Affymetrix GeneChip ${ }^{\circledR}$ Command Console ${ }^{\circledR}$ Software, the CEL files were analyzed using the Affymetrix Expression Console ${ }^{\mathrm{TM}}$ software v1.3.1 to obtain an intensity value signal for each probe set. The probesets were annotated using the Affymetrix annotation file from Netaffx (http://www.netaffx.com). Affymetrix array core probeset data was normalized with the Robust Multiarray Average (RMA) method. Transcripts with significant differential expression profiles were identified using the Significance Analysis of Microarray (SAM) algorithm (http://wwwstat.stanford.edu/ tibs/SAM/) with the Wilcoxon test and sample label permutations $(n=300)$ was used to identify genes of which expression varied significantly between one selected time point (day $0.5,1,2$ or 
3 ) and day 0 . Only transcripts with a fold change $(F C) \geq 2$ and significant false discovery rate $(F D R)<5 \%$ were retained. Hierarchical clustering was produced using the Cluster and Treeview softwares (PMID: 9843981). The gene ontology (GO) enrichment analysis, the biological processes and networks of differentially expressed genes were analyzed through the use of Ingenuity Pathway Analysis (QIAGEN Inc., https://www.qiagenbioinformatics.com/products/ingenuitypathway-analysis) (PMID: 24336805). Analysis of the expression of stemness and proliferation-related genes are based on a previously published data set from pluripotent stem cells in which a consensus stemness gene list ( $n=1076$ genes) was defined (PMID: 17204602) and from proliferating samples including rapidly dividing CD105+ endothelial cells, $\mathrm{CD} 71^{+}$early erythroid progenitors and cell lines originating from tumors in which a proliferation gene list was defined (PMID: 19128516). Venn diagrams were obtained using Venny tools and represent the number of genes in each comparison and the overlaps between the four comparison groups.

Microarray data are in accordance to Minimum Information About a Microarray Experiment (MIAME) guidelines.

\section{RT-qPCR analysis}

RNA was reverse transcribed using the Moloney Murine Leukaemia Virus Reverse Transcriptase (Invitrogen, ThermoFisher Scientific) in a non-gradient thermocycler. qPCR was then performed on $10 \mathrm{ng}$ of $\mathrm{CDNA}$ using SYBR ${ }^{\text {TM }}$ Green I PCR Master Mix (Roche Diagnostics, Meylan, France) or TaqMan ${ }^{\text {TM }}$ Universal Master Mix II, with UNG and specific primers (Tables 1-2). PCR reaction was performed as follow: $95^{\circ} \mathrm{C}$ for $5 \mathrm{~min} ; 40$ cycles at $95^{\circ} \mathrm{C}$ for $15 \mathrm{~s} ; 64^{\circ} \mathrm{C}$ for $10 \mathrm{~s}$ and $72^{\circ} \mathrm{C}$ for $20 \mathrm{~s}$ with SYBR Green or $95^{\circ} \mathrm{C}$ for $5 \mathrm{~min} ; 40$ cycles at $95^{\circ} \mathrm{C}$ for $15 \mathrm{~s}$ and $64^{\circ} \mathrm{C}$ for $10 \mathrm{~s}$ with TaqMan, in a ViiA 7 Real-Time PCR System (Applied Biosystems, ThermoFisher Scientific) and analyzed with the dedicated software. All values were normalized to the housekeeping gene RPS 9 and expressed as relative expression using the formulae $2^{-\Delta C t}$ or as fold change using the formulae $2^{-\Delta \Delta C t}$.

\section{Cell transfection}

BM-MSCs were transfected twice: 4 days ( $80 \%$ of confluence) and one day before inducing the chondrogenic differentiation. Transfection was done using $50 \mathrm{nM}$ of predesigned siNMB (TaqMan, siRNA ID: s9582; Applied Blosystems, Illkirch, France) or siRNA control (UAAGGCUAUGAAGAGAUACTT) using Oligofectamine $^{\text {TM }}$ in Opti-MEM ${ }^{\text {TM }}$ (Life Technologies SAS, Villebon-sur-Yvette, France) during 6 hours.

\section{Cell treatment}

BM-MSCs were treated with recombinant NMB (rNMB; peptide sequence: Gly-Asn-Leu-Trp-Ala-Thr-Gly-HisPhe-Mel- $\mathrm{NH}_{2}$; Sigma-Aldrich) for the 21 days of chondrogenesis. Two concentrations were evaluated: 100 or $1000 \mathrm{nM}$ and the medium was changed every 3-4 days. Passage 1 chondrocytes were plated at 60,000 cells/well of 6-well plates and treated with the NMBR antagonist BIM23042 (BIM; peptide sequence: DNal-Cys-Tyr-D-Trp-Lys-Val-Cys-Nal-NH 2 ; Tocris, Noyal Châtillon sur Seiche) at 50 nM during 3 days. 


\section{Western blot}

Micropellets were first washed with PBS, then stored in RIPA lysis buffer (Sigma Aldrich) containing a protease inhibitor cocktail (Sigma, P-8340) for $1 \mathrm{~h}$ at $-80^{\circ} \mathrm{C}$. Micropellets were then grinded and centrifuged at $12,000 \mathrm{rcf}$, at $4^{\circ} \mathrm{C}$ for $15 \mathrm{~min}$. Total proteins $(50 \mu \mathrm{g})$ diluted in Bolt LDS sample buffer and Bolt Sample Reducing Agent were heated at $70^{\circ} \mathrm{C}$ for 10 min and loaded on Bolt 4-12\% Bis-Tris Plus precast gels (Life Technologies). Samples were allowed to migrate for $30 \mathrm{~min}$ at $200 \mathrm{mV}$ using the MOPS SDS running buffer and transferred on nitrocellulose membranes using the iBlot $2 \circledR$ Gel Transfer Device (Life Technologies). Membranes were then blocked with $5 \%$ milk and probed with the anti-human NMB rabbit polyclonal primary antibodies (1:500; Cohesion Biosciences, Nanterre) at $4^{\circ} \mathrm{C}$, overnight. Antihuman Actin mouse polyclonal primary antibodies (1:5000) for $2 \mathrm{~h}$ at room temperature. After several rinses with tris phosphate buffered saline (TBS) containing 0.05\% tween-20 (Sigma-Aldrich), membranes were incubated with a horseradish peroxidase-conjugated goat anti-rabbit IgG antibody (1:50000) for $1 \mathrm{~h}$ at room temperature. Blots were revealed using a WesternBright ${ }^{\mathrm{TM}}$ Sirius Chemiluminescent detection kit (Advansta, Blagnac) and scanned (ChemiDoc XRS System, Biorad).

\section{Analysis of spontaneous calcium transients and calcineurin cellular activity}

Spontaneous calcium transients were monitored on micropellets at day 3 of differentiation. Micropellets were rinsed with buffer containing $145 \mathrm{mM} \mathrm{NaCl}, 5 \mathrm{mM} \mathrm{KCl}, 2 \mathrm{mM} \mathrm{CaCl}_{2}, 2 \mathrm{mM} \mathrm{MgCl}_{2}, 10 \mathrm{mM} \mathrm{HEPES}, 10$ $\mathrm{mM}$ glucose $(\mathrm{pH} 7.4)$ and incubated with Fluo 4-AM $(500 \mu \mathrm{g} / \mathrm{mL}$; Invitrogen) and Pluronic F-127 (0.1\%; Invitrogen) at $37^{\circ} \mathrm{C}$ for $45 \mathrm{~min}$. Calcium imaging was performed for 30 min using a Multi-photon Zeiss LSM 7MP OPO microscope (Zeiss, Marly le Roi, France) and analyzed with the ZEN software (Zeiss).

Calcineurin activity was measured using the calcineurin cellular activity assay kit (Enzo, Villeurbane, France). Day 3-micropellets were rinsed in Tris Buffered Saline (TBS), lysed in the lysis buffer containing the protease inhibitor cocktail and grinded using an Ultra-turrax dispenser. Samples were then stored at $-80^{\circ} \mathrm{C}$ before quantification of calcineurin cellular activity by measuring the $\mathrm{Ca}^{2+/}$ calmodulin-dependent Ser/Thr protein phosphatase 2B (PP2B) activity according to manufacturer's instructions.

\section{Statistical analysis}

Statistical analysis was performed using GraphPad Prism 7 software (San Diego, USA). Data were represented as mean \pm standard error of the mean (sem) of separate experiments. Data did not assume a Gaussian distribution and were analyzed using appropriate nonparametric statistical tests. The comparison between 2 unpaired groups was performed using a Mann-Whitney test. The comparison between one group compared to the control group normalized to 1 or $100 \%$ was performed using a Wilcoxon signed rank test. The comparison between different groups was analyzed by a Kruskall-Wallis test followed by a Dunn's multiple comparisons test. Correlations were analyzed with a nonparametric Spearman test. Statistical differences were indicated as $*$ : $p<0.05, * \star$ : $p<0.01, * \star *$ : $p<0.001$.

\section{Results}




\section{Global gene analysis in the early stages of chondrogenesis}

In order to identify new genes involved in the early steps of chondrogenesis, we performed a transcriptomic analysis on 3 independent samples of human BM-MSCs. BM-MSCs were induced to differentiate toward chondrocytes using the micropellet culture conditions in presence of TGF $\beta 3$. To minimize the modulation of gene expression induced by the culture of MSCs from 2D to 3D, we compared the genes expressed by micropellets in presence of TGF $\beta 3$ (chondrogenic condition) and in absence of TGF 33 (3D condition) at different time points (day 0, 0.5, 1, 2 and 3) (see the workflow in Fig. 1A). Chondrogenic differentiation of the three samples was confirmed at day 21 by increased expression of chondrocyte markers (data not shown). At each time point and in each sample, the gene expression was normalized to day 0 and compared between the two conditions (chondrogenic vs 3D). To validate our analysis, we examined the expression of three genes, TGFB3, TGF 1 and $S O X 9$ that are known to be increased at early stages of chondrogenesis. Quite similar gene expression kinetic profiles were obtained in the microarrays (upper panel) and by RT-qPCR analysis (bottom panel) for the three samples suggesting the validity of our transcriptomic data (Fig. 1B).

Using the Venny tool, we excluded the genes that were specific for and/or common to the 3D condition and found 1,929 genes that were specific for the chondrogenic condition and significantly modulated compared to day 0 . Global analysis of the genes that were differentially modulated at different time points as compared to day 0 revealed a higher number of genes up-regulated at day 0.5 and 1 and a higher number of genes down-regulated at day 2 and 3 (78\% of all modulated genes at day 3 ) (Fig. 1C). The number of up-regulated genes remained stable from day 1 to day 3 while the down-regulated genes constantly increased. Similar profiles were obtained for the two culture conditions (chondrogenic and 3D). Day 0.5 appeared as an essential time point because the up-regulated genes were only observed in the samples cultured in the chondrogenic condition. Interestingly, using a list of genes assigned to stemness or proliferation (see method section), we noticed that only a small proportion of modulated genes were involved in these processes suggesting that most of the genes might be related to the initiation of differentiation. For the following analyses, the genes common to the chondrogenic and 3D conditions were excluded as being related to the 3D culture and not to chondrogenesis.

Hierarchical clustering based on the transcripts differentially expressed in micropellets cultured under the chondrogenic condition highlighted a segregation of samples according to the time points: the very early modulated genes (days 0.5-1) and the early modulated genes (days 2-3) (Fig. 1D). Looking at the most highly regulated genes at the different time points, we identified many genes that were already known to be involved in chondrogenesis and/or expressed in chondrocytes according to the literature (data not shown). These genes were excluded and the remaining genes were attributed to subgroups. We classified a group of the most highly modulated genes (first 25 genes), or the group of very early genes (32 genes with a fold change value $>2$ ), or the group of early genes (13 genes with a fold change value $>2$ ) or the so-called interactive group, which included genes that were modulated in these last two groups and reported to interact in a same pathway, as determined using a Gene Ontology analysis. A Venn diagram encompassing the genes significantly modulated (with a p-value $<0.05$ ) summarized this analysis 
(Fig. 1E). Only one gene, ZFP36, was found expressed in the four groups while nine genes were expressed in three groups and twenty-two genes were common in two groups. At the end, twenty-four genes were retained as being specific for the chondrogenic condition and significantly modulated in at least two groups (Fig. 1F). Among the twenty-four genes selected, twenty-one genes were up-regulated and only three were down-regulated.

\section{Identification of NMB as specifically upregulated during chondrogenesis}

To validate the twenty-four genes selected from the transcriptomic analysis, six new samples of BMMSCs were induced to differentiate toward chondrocytes using the micropellet culture conditions in presence or in absence of TGF 33 . A kinetic study of mRNA expression during chondrogenesis revealed that eight genes (ETS2,PLAUR, NDN, NFKBIA, PRRG4, SPHK1, TNFAIP3, ZFP36) were not differentially modulated between the chondrogenic and 3D conditions (Fig. 2). The expression of three genes (KCNH1, $N R 4 A 1, N R G 1)$ was significantly decreased compared to day 0 whatever the culture conditions. Ten genes (PDK4, FKBP5, HEYL, ITPR1, KCNE4, JARID2, PFKFB3, SLC19A2, TIPARP, RASD2) were significantly upregulated compared to day 0 but with a fold change less than 10 or not significantly different from the 3D condition. Finally, three genes (RGCC, TSPAN2, NMB) were selected because they were up-regulated more than 40 -fold at days 0.5 or 1 and their expression was significantly higher in the chondrogenic condition than in the $3 \mathrm{D}$ condition in at least one-time point.

The specificity of expression of these factors during chondrogenesis was evaluated on 3 independent samples of BM-MSCs induced to differentiate toward adipocytes or osteoblasts. After 21 days in adipogenic medium, the adipocyte markers ( $P P A R Y, F A B P 4, L P L)$ were significantly up-regulated and MSCs were stained by Oil red $O$ indicating the storage of triglycerides in lipid droplets (Fig. 3A). No differentiation occurred in the proliferation medium. At different time points during adipogenesis, the expression of the three genes was quantified. The expression of RGCC was significantly increased at days 7 and 10 while the expression of TSPAN2 was increased after day 7 only in the proliferation condition (Fig. 3B). The expression of $N M B$ did not change during the course of adipogenesis. After 21 days in osteoblastogenic medium, the osteogenic markers ( $B G L A P, R U N X 2, A L P L)$ were significantly upregulated and mineralization was demonstrated by Alizarin Red S staining (Fig. 3C). During osteoblastogenesis, the expression of RGCC increased by more than 200-fold from day 4 till day 21 while the expression of TSPAN2 and NMB was not modulated (Fig. 3D). Altogether, we found that the two genes TSPAN2 and NMB were specifically modulated during chondrogenesis only. TSPAN2 is a plasma membrane associated protein while $N M B$ is a secreted factor. We therefore focused our attention on $N M B$, whose soluble form might allow to induce chondrogenesis in a more controllable way.

\section{Correlation between the expression of NMB and chondrocyte markers}

We investigated the expression of $\mathrm{NMB}$ at the protein level during chondrogenesis and found a higher amount of NMB at day 3 compared to day 0 , confirming its upregulation at an early chondrogenic stage (Fig. 4A). We then determined whether $N M B$ expression was differentially regulated in OA MSC samples, for which chondrogenesis is known to be impaired. We showed that $N M B$ and its receptor $N M B R$ were 
expressed at low but similar levels in BM-MSCs with no difference when BM-MSCs were isolated from healthy or OA donors (Fig. 4B). BM-MSC samples from both healthy and OA subjects were then induced to differentiate into chondrocytes that expressed all the specific markers SOX9, COL2A1 variant $B$, COL 10A1, and ACAN after 21 days (Fig. 4C). Interestingly, the expression of $N M B$ was upregulated at early time points of chondrogenesis (from day 0.5 to 3 ) in BM-MSCs independently of the origin of BMMSCs, healthy or OA. We also found out that the basal expression of $N M B$ in BM-MSCs at day 0 was correlated with the expression level of all tested chondrocyte markers SOX9, COL2B, ACAN, COL 1OA1 at day 21 (Fig. 4D). Similar correlation was found between NMB and chondrocyte markers at day 3 but no correlation between $N M B$ and adipogenic or osteogenic markers was observed (data not shown). We therefore confirmed the upregulation of $N M B$ during the first 3 days of chondrogenesis and reported its prognostic value for high chondrogenic differentiation capacity of BM-MSCs.

\section{Expression of NMB is required for chondrogenesis}

Because NMB expression is correlated with high differentiation capacity, we tested the impact of exogenously added recombinant NMB (rNMB) on the differentiation of BM-MSCs. Compared to control BM-MSCs cultured in micropellets with TGF $\beta 3$, the addition of increasing doses of rNMB did not modulate the expression of chondrocyte markers (SOX9, COL2A1 variant $\mathrm{B}$, and $A C A N$ ) by day 21 (Fig. 5A). We checked whether the lack of inductive effect of rNMB could be related to a possible role on proliferation. The addition of rNMB (100 or $1000 \mathrm{nM}$ ) during the expansion of BM-MSCs for 5 days did not impact their proliferation compared to the untreated control (Fig. 5B). We then evaluated whether the down-regulation of NMB could interfere with TGF $\beta 3$-induced chondrogenesis, using a RNA interference approach. BM-MSCs were transfected twice with a siRNA targeting NMB (siNMB) or a control siRNA (siCT). The transfection of siNMB inhibited the expression of $N M B$ by $80 \%$ and $77 \%$ at day 0 and day 3 , respectively (Fig. 5C). Macroscopically at day 3, the micropellets obtained with siNMB-transfected BMMSCs did not form a homogenous round/ovoid shape and were more loosen than those from control cells (Fig. 5D). The down-regulation of NMB was likely not related to an inhibitory effect on MSC proliferation (Fig. 5E) but we observed a significant inhibition of the adhesion molecule CDH2 in siNMBtransfected BM-MSCs (Fig. 5F). No impact on the expression of other adhesion molecules ITGav, ITG 1 and ITG $\beta 5$ was noticed. Interestingly, the downregulation of NMB resulted in the downregulation of the chondrocyte markers $S O X 9, C O L 2 A 1$ variant $B, A C A N$ and $C O L 10 A 1$ at day 21 , although significance was reached only for SOX9 and COL2A1 variant B (Fig. 5G). These results indicated that NMB acts in an autocrine manner in MSCs and its expression is necessary but not sufficient for the induction of chondrogenic differentiation.

\section{NMB regulates Sox9 expression through PLC signaling and calcium release at early steps of chondrogenesis}

Multiple intracellular signaling pathways are activated upon binding of NMB to its receptors. One of these includes the hydrolysis of phosphatidyl inositol 4,5-biphosphate $\left(\mathrm{PIP}_{2}\right)$ by the isoform $\beta$ of phospholipase $\mathrm{C}\left(\mathrm{PLC}_{\beta}\right)$ into 1,2-diacylglycerol (DAG) and inositol 1,4,5-triphosphate $\left(\mathrm{iP}_{3}\right)$. Binding of $\mathrm{iP}_{3}$ to its receptors 
in the endoplasmic reticulum triggers the release of $\mathrm{Ca}^{2+}$ in the cytosol [19]. Because the increase of $\mathrm{Ca}^{2+}$ influx was previously reported to be indispensable for proper chondrogenesis [20], we investigated the intracellular calcium concentration in siNMB-transfected MSCs induced to differentiate toward chondrocytes. The spontaneous calcium oscillations were determined in Fluo-4-AM loaded BM-MSCs at day 3 of differentiation when NMB levels were high. A representative time course of maximal $\mathrm{Ca}^{2+}$ intensity revealed both a lower number and a lower maximal intensity of $\mathrm{Ca}^{2+}$ flux pics in micropellets of siNMB-transfected BM-MSCs compared to control micropellets (Fig. 6A). This result was confirmed by quantifying the $\mathrm{Ca}^{2+}$ responsive rate, which was lower in micropellets of siNMB-transfected BM-MSCs (Fig. 6B). We also determined the levels of cellular calcineurin phosphatase activity, which is dependent of $\mathrm{Ca}^{2+}$ concentration and positively regulates chondrogenesis [20]. At day 3 of chondrogenesis, the calcineurin activity was significantly reduced in micropellets of siNMB-transfected BM-MSCs compared to siCT-transfected BM-MSCs, which was related with the inhibition of SOX9 expression (Fig. 6C-D). Taken together, these results suggest that NMB is the key player of intracytoplasmic $\mathrm{Ca}^{2+}$ regulation resulting in successful chondrogenesis.

\section{Discussion}

With 3 biologic replicates and four time points during the first 3 days of chondrogenic differentiation of MSCs, our data presents an in-depth analysis of the gene expression changes induced during MSC chondrogenesis. The analysis provided a list of genes already known to be modulated during chondrogenesis (data not shown) and a set of genes not previously described as associated with chondrogenesis. The main difference of our analysis with other large-scale transcriptomic analyses is the identification of genes that were modulated during the chondrogenic differentiation of MSCs, but not related to the changes from 2D to 3D culture conditions $[11,13,14]$. This allowed to identify several genes that might be important regulators of chondrogenesis. We focused our attention on NMB, a secreted factor that might be clinically used as an exogenous agent for MSCs differentiation, without genetic engineering.

The importance of the intracellular $\mathrm{Ca}^{2+}$ modulation at the onset of chondrogenic differentiation has been shown using high-density cultures of chicken limb bud-derived mesenchymal cells [20]. In this study, the basal level of $\mathrm{Ca}^{2+}$ was reported to increase on day 3 of differentiation. The changes of cytosolic free $\mathrm{Ca}^{2+}$ concentration were not dependent on intracellular $\mathrm{Ca}^{2+}$ stores but rather largely dependent on extracellular sources. Interestingly, slight and transient elevation of cytosolic $\mathrm{Ca}^{2+}$ levels promoted differentiation through the regulation of cell proliferation while high increase of $\mathrm{Ca}^{2+}$ inhibited chondrogenesis. In line with our results where NMB is transiently upregulated during the first days of chondrogenesis, the authors demonstrated that transient elevation of $\mathrm{Ca}^{2+}$ preceded differentiation and manipulation of intracellular concentration of calcium altered chondrogenesis. The signaling molecule in this process was calcineurin, whose role in the regulation of intracellular $\mathrm{Ca}^{+}{ }^{+}$influx was crucial. A previous study described the positive role of calcineurin in the phosphorylation of SOX9 and 
chondrogenesis regulation [21]. $\mathrm{Ca}^{2+}$ influx-dependent SOX9 activation was also reported during chondrogenesis of C3H10T1/2 MSCs and ATDC5 chondroprogenitor cells [22]. In these cells, the activation of SOX9 by transient receptor potential vanilloid 4 (TRPV4), a calcium channel involved in $\mathrm{Ca}^{2+} /$ calmodulin signaling induced GAG accumulation when applied with other inductive signals such as insulin or BMP-2 and contributed to the process of chondrogenesis. Other types of calcium channels, the voltage gated $\mathrm{Ca}^{2+}$ channels (VGCC) are also key regulators in chondrocytes. Of interest, $\mathrm{Ca}^{2+}$ influx via the $\mathrm{Ca}_{\mathrm{V}} 3.2 \mathrm{VGCC}$ activates the calcineurin/nuclear factor of the activated T-cell (NFAT) signaling pathway and a NFAT binding site has been identified within the SOX9 promoter [23]. Different types of calcium channels have been identified in chondrocytes, including VGCC, TRP, acid-sensing ion channels as discussed in a recent review on the chondrocyte channelome [24]. The role of these channels in chondrogenesis is still imperfectly understood. However, the importance of $\mathrm{Ca}^{2+}$ influx from the extracellular compartment and the activation of the calcineurin/NFAT signaling pathway leading to SOX9 activation has been demonstrated in chondrocytes [20].

In addition to extracellular $\mathrm{Ca}^{2+}$ influx through plasma membrane associated channels, transient increase of cytosolic $\mathrm{Ca}^{2+}$ can occur through the release of $\mathrm{Ca}^{2+}$ from endoplasmic reticulum (ER) stores. Rapid depletion of $\mathrm{ER} \mathrm{Ca}^{2+}$ stores activates $\mathrm{Ca}^{2+}$ entry across the plasma membrane, a process called storeoperated $\mathrm{Ca}^{2+}$ entry (SOCE), which has been reported in chondrocytes and chondroprogenitor cells [20, 25]. In this process, phospholipase $C$ (PLC) increases inositol phosphate levels and induces cytosolic release of intracellular $\mathrm{Ca}^{2+}$ from ER stores. Of interest, the PLC signaling pathway is activated by NMB upon binding to its receptors in various NMBR-expressing cells [19]. The signaling cascade results in DNA synthesis and cell proliferation in a number of tumor cells [26, 27]. In MSCs, the modulation of NMB expression did not impact their proliferation but the inhibition of $\mathrm{NMB}$ resulted in lower $\mathrm{Ca}^{2+}$ and calcineurin activity, and reduced SOX9 expression. We therefore suggest that NMB-induced intracellular $\mathrm{Ca}^{2+}$ influx induces SOX9-dependent chondrogenesis through the activation of calcineurin/NFAT pathway, probably via PLC activation (Fig. 7). These results provide a novel insight on the regulation of $\mathrm{Ca}^{2+}$ signaling pathway during chondrogenesis.

Besides their role during chondrogenesis, $\mathrm{Ca}^{2+}$ signaling pathways are important components of mechanotransduction pathways in chondrocytes. Indeed, a positive correlation between cartilage extracellular matrix synthesis and $\mathrm{Ca}^{2+}$ signaling has been reported under mechanical load [28]. TRPV4 is a positive regulator of SOX9 and mutations in this gene are linked to chondrodysplasia [29]. In addition, TRPV6-knockout mice exhibit severe OA changes suggesting that TRPV6 may be a chondroprotective factor [30]. Accumulating evidence indicates that altered expression or function of $\mathrm{Ca}^{2+}$ channels, including TRP channels, might be implicated in OA development [24]. An increase in $\mathrm{Ca}^{2+}$ signaling may be one cause of chondrocyte dysfunction in OA suggesting that inhibition of $\mathrm{Ca}^{2+}$ channels could be therapeutic targets for OA [31]. Actually, the deletion of TRPV4 reduced the severity of age-related OA development although it did not prevent destabilization of the medial meniscus (DMM)-induced OA development [32]. Our preliminary results indicate that down-regulation of NMB using a siRNA approach 
in chondrocytes from OA patients results in the up-regulation of chondrocyte markers while the upregulation of NMB using the NMBR inhibitor BIM reverses this effect (supl. Fig. 1). These results confirmed that the increase of free cytosolic $\mathrm{Ca}^{2+}$ above a threshold level could be detrimental to chondrocyte homeostasis and chondrogenesis [20]. In line with these data, a pilot study in OA patients has revealed that $\mathrm{Ca}^{2+}$ antagonists may exert beneficial side clinical effects in preventing the progression of the disease [33]. These results therefore suggest that modulation of $\mathrm{Ca}^{2+}$ signaling may counteract the catabolic response and defective synthesis of extracellular matrix molecules in OA cartilage. A better understanding of the involvement of $\mathrm{Ca}^{2+}$ channels and the modulation of $\mathrm{Ca}^{2+}$ signaling in chondrocytes under healthy and OA conditions is needed before to investigate novel therapeutic strategies.

\section{Conclusion}

In the present study, we highlighted an inducting role of NMB in the early phases of MSC differentiation towards chondrocytes that has not been previously described. We made the link between the transient upregulation of $\mathrm{NMB}$ during the first days of chondrogenesis and the modulation of $\mathrm{Ca}^{2+}$ signaling pathway that is essential for optimal differentiation.

\section{Abbreviations}

bFGF: basic fibroblast growth factor

BM: bone marrow

CDKN2D: cyclin-dependent kinase 4 inhibitor 2D

DAG: 1,2-diacylglycerol

ECM: extracellular matrix

FC: fold change

FCS: fetal calf serum

FDR: false discovery rate

GO: gene ontology

$\mathrm{iP}_{3}$ : inositol 1,4,5-triphosphate

MIAME: Minimum Information About a Microarray Experiment

MSC: mesenchymal stromal/stem cell 
NFAT: calcineurin/nuclear factor of the activated T-cell

NMB: neuromedin

$\mathrm{OA}$ : osteoarthritis

$\mathrm{PIP}_{2}$ : phosphatidyl inositol 4,5-biphosphate

PLC: phospholipase C

SAM: Significance Analysis of Microarray

Sem: standard error of the mean

siCT: control siRNA

siNMB: siRNA targeting NMB

SOCE: store-operated $\mathrm{Ca}^{2+}$ entry

TGF $\beta$ : transforming growth factor $\beta$

TGM2: transglutaminase 2

TRPV4: transient receptor potential vanilloid 4

VGCC: voltage gated $\mathrm{Ca}^{2+}$ channels

\section{Declarations}

\section{Ethics approval and consent to participate}

Human MSCs and chondrocytes were recovered from patients after written informed consent as approved by the French Ministry of Higher Education and Research (DC-2009-1052).

\section{Consent for publication}

Informed consent for publication has been received.

\section{Availability of data and materials}

Gene expression data from this study have been deposited at Gene Expression Omnibus.

\section{Competing interests}

The authors disclose any conflict of interest. 
Acknowledgements

Not applicable

\section{Funding}

Authors would like to acknowledge funding support from the Inserm Institute, the University of Montpellier. We thank Servier for financial support of this project.

\section{Author's contributions}

Marie Maumus performed research, analyzed data and wrote the paper; Guillaume Fonteneau, Maxime Ruiz performed research and analyzed data; Hassan Boukhaddaoui performed research; Said Assou, Frédéric De Ceuninck, Philippe Pastoureau and Christian Jorgensen analyzed data; Danièle Noël designed research, analyzed data and wrote the paper.

\section{References}

1. Vinatier $C$, et al. Cartilage engineering: a crucial combination of cells, biomaterials and biofactors. Trends Biotechnol. 2009;27:307-314.

2. Martin AR, et al. Emerging therapies for cartilage regeneration in currently excluded 'red knee' populations. NPJ Regen Med. 2019;4:12.

3. Green JD, et al. Multifaceted signaling regulators of chondrogenesis: Implications in cartilage regeneration and tissue engineering. Genes Dis. 2015;2:307-327.

4. Griffiths R, et al. The Transcription Factor-microRNA Regulatory Network during hESCchondrogenesis. Sci Rep. 2020;10:4744.

5. Stelcer E, et al. The Role of MicroRNAs in Early Chondrogenesis of Human Induced Pluripotent Stem Cells (hiPSCs). Int J Mol Sci. 2019;20.

6. Yang Z, et al. MicroRNA expression profiles in human adipose-derived stem cells during chondrogenic differentiation. Int J Mol Med. 2015;35:579-586.

7. Bakhshandeh B, et al. A microRNA signature associated with chondrogenic lineage commitment. J Genet. 2012;91:171-182.

8. Wang $L$, et al. Long noncoding RNAs expression signatures in chondrogenic differentiation of human bone marrow mesenchymal stem cells. Biochem Biophys Res Commun. 2015;456:459-464.

9. Guerit D, et al. Sox9-regulated miRNA-574-3p inhibits chondrogenic differentiation of mesenchymal stem cells. PLoS One. 2013;8:e62582.

10. Djouad F, et al. Transcriptomic analysis identifies Foxo3A as a novel transcription factor regulating mesenchymal stem cell chrondrogenic differentiation. Cellular reprogramming. 2009;11:407-416.

11. Huynh NPT, et al. High-depth transcriptomic profiling reveals the temporal gene signature of human mesenchymal stem cells during chondrogenesis. FASEB J. 2019;33:358-372. 
12. Mathieu $\mathrm{M}$, et al. Involvement of angiopoietin-like 4 in matrix remodeling during chondrogenic differentiation of mesenchymal stem cells. J Biol Chem. 2014;289:8402-8412.

13. Skreti G, et al. Temporal and spatial patterns of gene profiles during chondrogenic differentiation. IEEE J Biomed Health Inform. 2014;18:799-809.

14. Somoza RA, et al. Transcriptome-Wide Analyses of Human Neonatal Articular Cartilage and Human Mesenchymal Stem Cell-Derived Cartilage Provide a New Molecular Target for Evaluating Engineered Cartilage. Tissue Eng Part A. 2018;24:335-350.

15. Georgi N, et al. MicroRNA Levels as Prognostic Markers for the Differentiation Potential of Human Mesenchymal Stromal Cell Donors. Stem Cells Dev. 2015;24:1946-1955.

16. Kanawa M, et al. Genetic Markers Can Predict Chondrogenic Differentiation Potential in Bone Marrow-Derived Mesenchymal Stromal Cells. Stem Cells Int. 2018;2018:9530932.

17. Johnstone $B$, et al. In vitro chondrogenesis of bone marrow-derived mesenchymal progenitor cells. Exp Cell Res. 1998;238:265-272.

18. Maria AT, et al. Human adipose mesenchymal stem cells as potent anti-fibrosis therapy for systemic sclerosis. J Autoimmun. 2016;70:31-39.

19. Ohki-Hamazaki H. Neuromedin B. Prog Neurobiol. 2000;62:297-312.

20. Matta $\mathrm{C}$, et al. Cytosolic free $\mathrm{Ca} 2+$ concentration exhibits a characteristic temporal pattern during in vitro cartilage differentiation: a possible regulatory role of calcineurin in Ca-signalling of chondrogenic cells. Cell Calcium. 2008;44:310-323.

21. Zakany R, et al. Hydrogen peroxide inhibits formation of cartilage in chicken micromass cultures and decreases the activity of calcineurin: implication of ERK1/2 and Sox9 pathways. Exp Cell Res. 2005;305:190-199.

22. Muramatsu S, et al. Functional gene screening system identified TRPV4 as a regulator of chondrogenic differentiation. J Biol Chem. 2007;282:32158-32167.

23. Lin SS, et al. Cav3.2 T-type calcium channel is required for the NFAT-dependent Sox 9 expression in tracheal cartilage. Proc Natl Acad Sci U S A. 2014;111:E1990-1998.

24. Mobasheri A, et al. The chondrocyte channelome: A narrative review. Joint Bone Spine. 2019;86:2935.

25. Fodor J, et al. Store-operated calcium entry and calcium influx via voltage-operated calcium channels regulate intracellular calcium oscillations in chondrogenic cells. Cell Calcium. 2013;54:1-16.

26. Lach EB, et al. Mitogenic signaling by transfected neuromedin B receptors in Rat- 1 cells. Cell Growth Differ. 1995;6:1427-1435.

27. Moody TW, et al. Neuromedin B stimulates arachidonic acid release, c-fos gene expression, and the growth of C6 glioma cells. Peptides. 1995;16:1133-1140.

28. Weber JF, Waldman SD. Calcium signaling as a novel method to optimize the biosynthetic response of chondrocytes to dynamic mechanical loading. Biomech Model Mechanobiol. 2014;13:1387-1397. 
29. Leddy HA, et al. Unraveling the mechanism by which TRPV4 mutations cause skeletal dysplasias. Rare Dis. 2014;2:e962971.

30. Song $T$, et al. Regulation of chondrocyte functions by transient receptor potential cation channel V6 in osteoarthritis. J Cell Physiol. 2017;232:3170-3181.

31. Berridge MJ. The Inositol Trisphosphate/Calcium Signaling Pathway in Health and Disease. Physiol Rev. 2016;96:1261-1296.

32. O'Conor CJ, et al. Cartilage-Specific Knockout of the Mechanosensory lon Channel TRPV4 Decreases Age-Related Osteoarthritis. Sci Rep. 2016;6:29053.

33. Daniilidis K, et al. Positive side effects of $\mathrm{Ca}$ antagonists for osteoarthritic joints-results of an in vivo pilot study. J Orthop Surg Res. 2015;10:1.

\section{Tables}

Table 1: List of primers for SYBR Green based PCR 


\begin{tabular}{|c|c|c|}
\hline Gene name & Sequence forward & Sequence reverse \\
\hline$A C A N$ & TCGAGGACAGCGAGGCC & TCGAGGGTGTAGCGTGTAGAGA \\
\hline$A L P L$ & CCACGTCTTCACATTTGGTG & GCAGTGAAGGGCTTCTTGTC \\
\hline$B G L A P$ & GGCGCTACCTGTATCAATGG & TCAGCCAACTCGTCACAGTC \\
\hline $\mathrm{CDH} 2$ & CCTCCAGAGTTTACTGCCATGAC & GATGGATCTCCGCCACTGATTC \\
\hline COL1A1 & CCTGGATGCCATCAAAGTCT & CGCCATACTCGAACTGGAAT \\
\hline COL2A1varB & CAGACGCTGGTGCTGCT & TCCTGGTTGCCGGACAT \\
\hline COL3A1 & CGCCCTCCTAATGGTCAAGG & AGGGCCTGAAGGACCAGCTT \\
\hline COL 10A1 & TGCTGCCACAAATACCCTTT & GTGGACCAGGAGTACCTTGC \\
\hline FABP4 & ATGGGATGGAAAATCAACCA & GTGGAAGTGACGCCTTTCAT \\
\hline ITGav & AACTCAAGCAAAAGGGAGCA & GGGTTGCAAGCCTGTTGTAT \\
\hline ITG $\beta 1$ & TGATGCAGTACTCCAGGCAG & AATCTCCACCGTTGTTCCAG \\
\hline ITG $\beta 5$ & GCCTTTCTGTGAGTGCGACAAC & CCGATGTAACCTGCATGGCACT \\
\hline$L P L$ & GTCCGTGGCTACCTGTCATT & TGGATCGAGGCCAGTAATTC \\
\hline MMP13 & GACTTCCCAGGAATTGGTGA & TACCCCAAATGCTCTTCAGG \\
\hline NMBR & GAAACCCCCACGGAAGTGAG & GGAGTTGGTGATCCGCTGTG \\
\hline PPARY & CCAGAAAGCGATTCCTTCAC & TGCAACCACTGGATCTGTTC \\
\hline RSP9 & ATGAAGGACGGGATGTTCAC & GATTACATCCTGGGCCTGAA \\
\hline RunX2 & CGGAATGCCTCTGCTGTTAT & TTCCCGAGGTCCATCTACTG \\
\hline Sox9 & AGGTGCTCAAAGGCTACGAC & GTAATCCGGGTGGTCCTTCT \\
\hline
\end{tabular}

Table 2: List of primers for TaqMan based PCR 


\begin{tabular}{|ll|}
\hline Gene name & Primer ID \\
\hline ETS2 & Hs01036305_m1 \\
\hline FKBP5 & Hs01561006_m1 \\
\hline HEYL & Hs00232718_m1 \\
\hline ITPR1 & Hs00181881_m1 \\
\hline JARID2 & Hs01004460_m1 \\
\hline KCNE4 & Hs00298953_m1 \\
\hline KCNH1 & Hs00924320_m1 \\
\hline NDN & Hs00267349_s1 \\
\hline NFKBIA & Hs00153283_m1 \\
\hline NMB & Hs00963142_m1 \\
\hline NR4A1 & Hs00374226_m1 \\
\hline NRG1 & Hs00247620_m1 \\
\hline PDK4 & Hs01037712_m1 \\
\hline PFKFB3 & Hs00998700_m1 \\
\hline PLAUR & Hs00958880_m1 \\
\hline PRRG4 & Hs00225378_m1 \\
\hline RASD2 & Hs00374755_m1 \\
\hline RGCC & Hs00204129_m1 \\
\hline RPS9 & Hs02339424_m1 \\
\hline SLC19A2 & Hs00949693_m1 \\
\hline SPHK1 & Hs01116530_g1 \\
\hline TIPARP & Hs00296054_m1 \\
\hline TNFAIP3 & Hs00234713_m1 \\
\hline TSPAN2 & Hs00194836_m1 \\
\hline ZFP36 & Hs00185658_m1 \\
\hline
\end{tabular}

Figures 

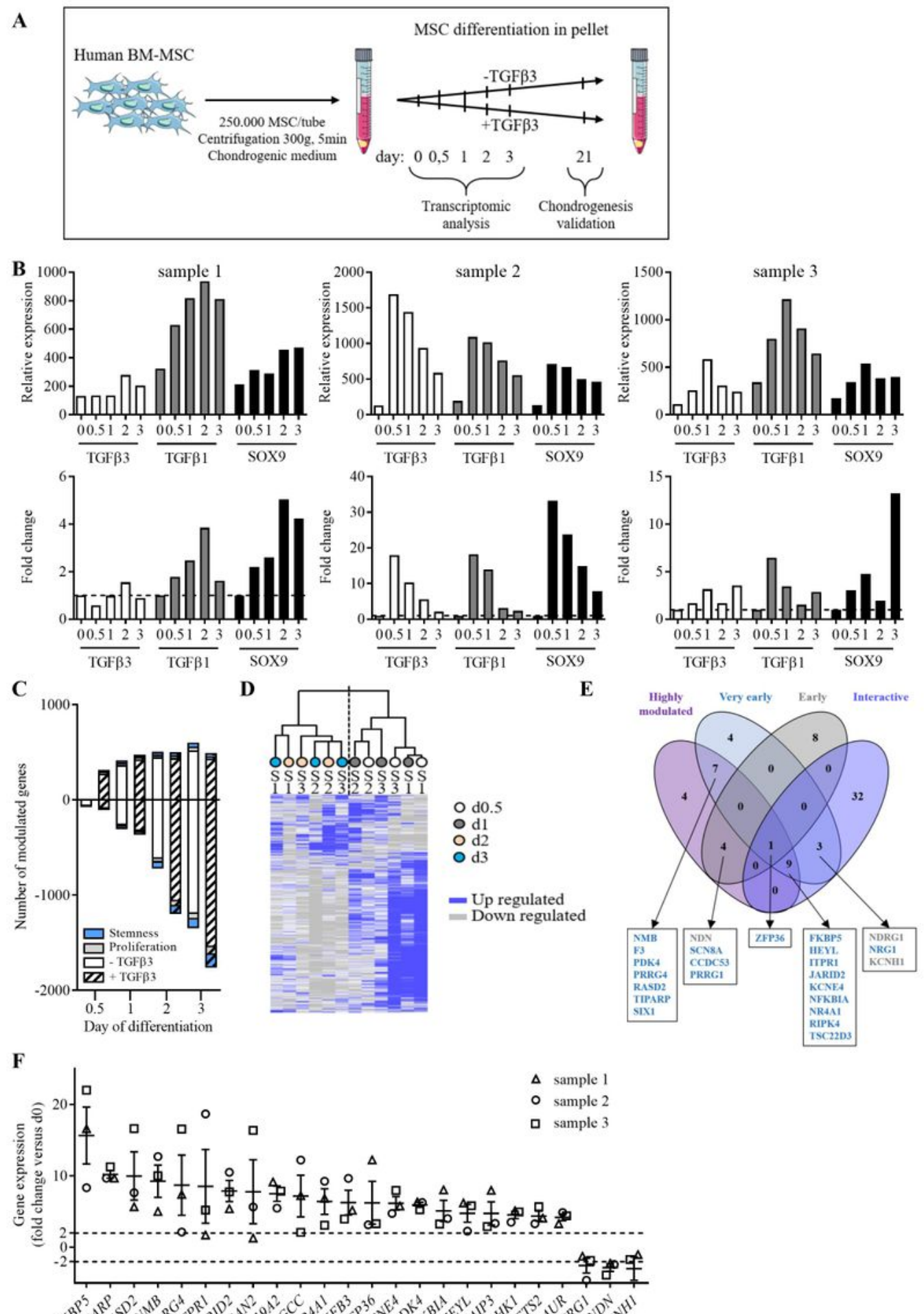

Down regulated

स

\section{Figure 1}

Microarray analysis and gene selection. a Experimental work flow. Bone-marrow MSCs (BM-MSC) were induced to differentiate into chondrocytes using the pellet culture conditions with or without TGF $\beta 3$.

Pellets were recovered at different time points (day 0, 0.5, 1, 2 and 3) for transcriptomic analysis and at $\mathrm{d} 21$, for validating chondrogenic differentiation. b Fold change expression of 3 selected genes in the 3 samples used in microarrays as determined by the transcriptomic analysis (upper panel) or by real-time 
qPCR (bottom panel). c Number of significantly up-regulated (positive values) and down-regulated (negative values) genes at different time points as compared to d0, in samples differentiated without (blank bar) or with TGFß3 (hatched bar). The number of genes relative to proliferation or stemness signature are in grey and blue, respectively. $d$ Unsupervised hierarchical clustering. Gene expression for the 3 BM-MSC samples (S1-3) cultured in TGFB3 condition, at different time points ( $d 0.5,1,2,3)$ using the 17000 probe sets. Up regulated and down regulated genes are in blue and grey colors, respectively. $\mathrm{e}$ Venn diagram including the top 25 up- and down-regulated genes independently of the time points (called "most regulated", in purple), the genes significantly regulated at d0.5 and d1 (called "very early genes", in blue), the genes significantly regulated at $d 2$ and $d 3$ (called "early genes", in grey) and the genes significantly regulated at the 4 time points and interacting within the same pathway as determined by IPA analysis (called "interactive", in dark purple). Up- and down-regulated selected genes are in blue and grey, respectively. f Relative expression of the genes selected in E) as determined by the transcriptomic analysis. 

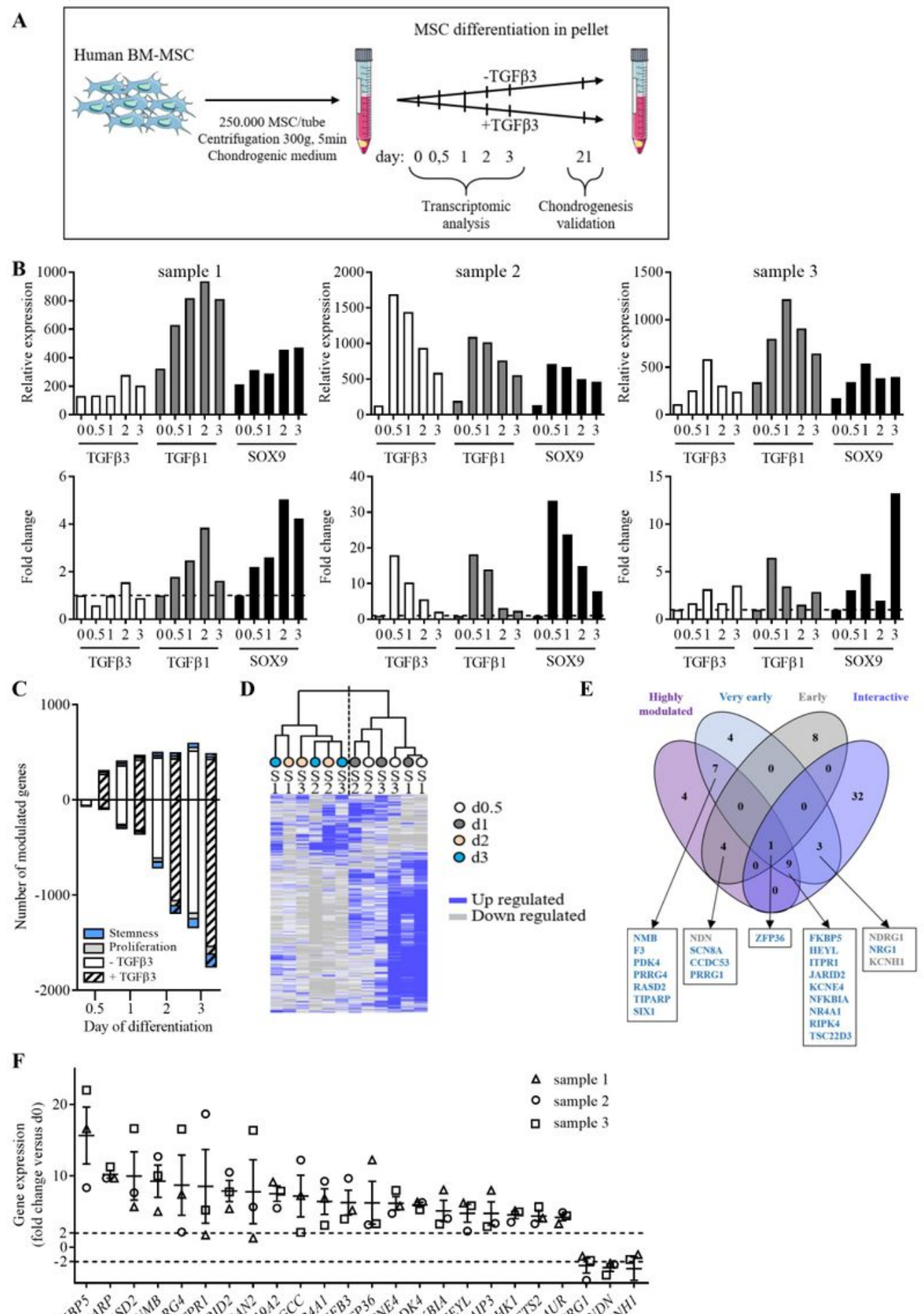

Down regulated

स

\section{Figure 1}

Microarray analysis and gene selection. a Experimental work flow. Bone-marrow MSCs (BM-MSC) were induced to differentiate into chondrocytes using the pellet culture conditions with or without TGF $\beta 3$.

Pellets were recovered at different time points (day 0, 0.5, 1, 2 and 3) for transcriptomic analysis and at $\mathrm{d} 21$, for validating chondrogenic differentiation. b Fold change expression of 3 selected genes in the 3 samples used in microarrays as determined by the transcriptomic analysis (upper panel) or by real-time 
qPCR (bottom panel). c Number of significantly up-regulated (positive values) and down-regulated (negative values) genes at different time points as compared to d0, in samples differentiated without (blank bar) or with TGFß3 (hatched bar). The number of genes relative to proliferation or stemness signature are in grey and blue, respectively. $d$ Unsupervised hierarchical clustering. Gene expression for the 3 BM-MSC samples (S1-3) cultured in TGFB3 condition, at different time points ( $d 0.5,1,2,3)$ using the 17000 probe sets. Up regulated and down regulated genes are in blue and grey colors, respectively. $\mathrm{e}$ Venn diagram including the top 25 up- and down-regulated genes independently of the time points (called "most regulated", in purple), the genes significantly regulated at d0.5 and d1 (called "very early genes", in blue), the genes significantly regulated at $d 2$ and $d 3$ (called "early genes", in grey) and the genes significantly regulated at the 4 time points and interacting within the same pathway as determined by IPA analysis (called "interactive", in dark purple). Up- and down-regulated selected genes are in blue and grey, respectively. f Relative expression of the genes selected in E) as determined by the transcriptomic analysis. 

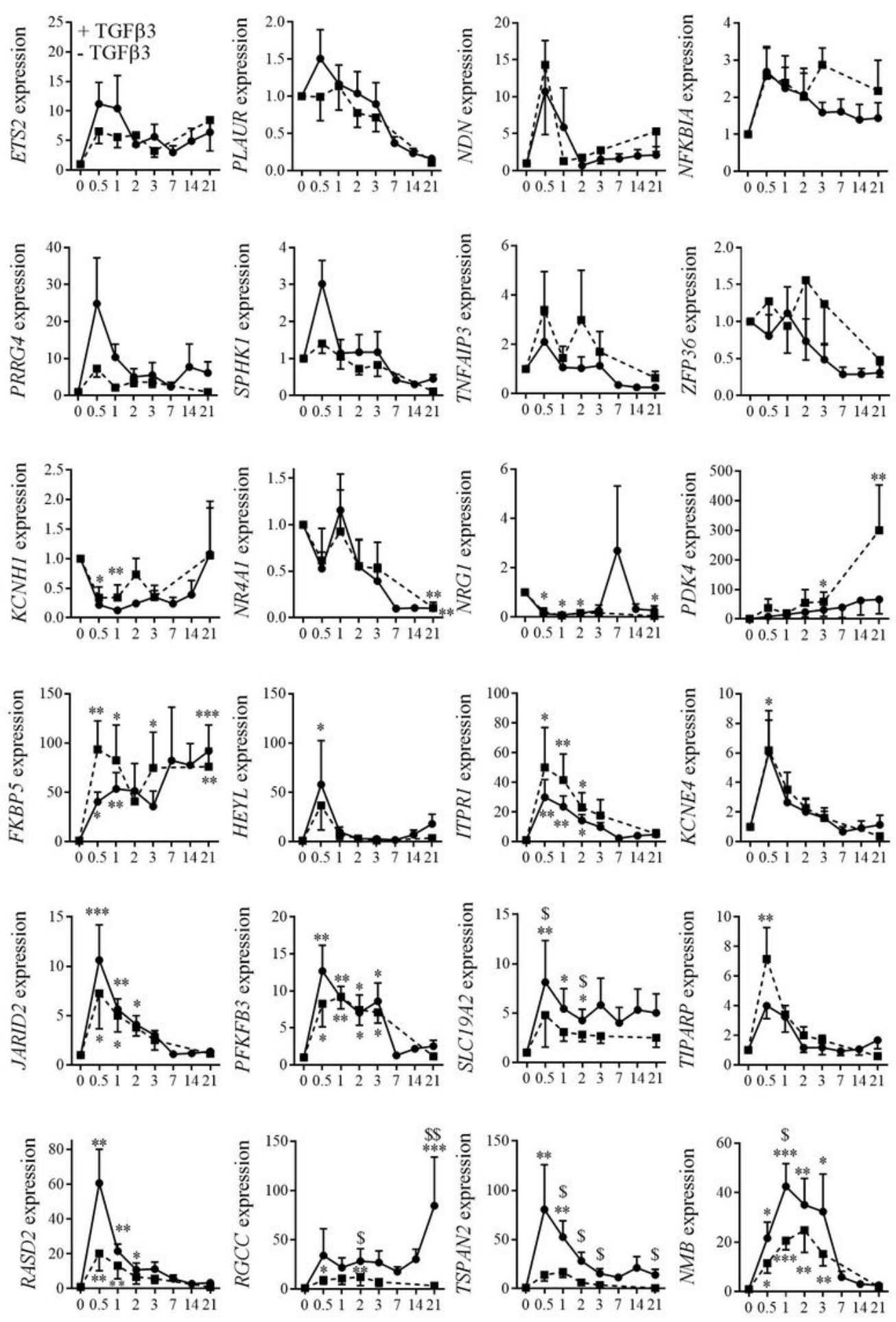

\section{Figure 2}

Expression kinetics of selected genes during chondrogenesis. Expression of selected genes were quantified at different time point in 6 individual BM-MSC replicates during chondrogenesis in pellet conditions with (black line) or without TGF 33 (hatched line) by RT-qPCR. Results are normalized to the expression at day 0 and expressed as the mean \pm sem. Statistical significance of gene expression was indicated as $*: p \leq 0.05, * *: p \leq 0.01, * * *: p \leq 0.001$ when compared to d0 and as $\$: p \leq 0.05, \$ \$: p \leq 0.01$ 
when samples cultured + or - TGF 33 were compared. (HEYL: Hes Related Family BHLH Transcription Factor with YRPW Motif Like; PDK4 (Pyruvate Dehydrogenase Kinase 4); TIPARP (TCDD Inducible Poly(ADP-Ribose) Polymerase); NDN (Necdin, MAGE Family Member); ZFP36 (ZFP36 Ring Finger Protein); TNFAIP3 (TNF Alpha Induced Protein); KCNH1 (Potassium Voltage-Gated Channel Subfamily H Member 1); FKBP5 (FKBP Prolyl Isomerase 5); PRRG4 (Proline Rich And Gla Domain;) ETS2 (ETS ProtoOncogene 2, Transcription Factor); JARID2 (Jumonji And AT-Rich Interaction Domain Containing 2); ITPR1 (Inositol 1,4,5-Trisphosphate Receptor Type 1); SLC19A2 (Solute Carrier Family 19 Member 2); NRG1 (Neuregulin 1); PFKFB3 (6-Phosphofructo-2-Kinase/Fructose-2,6-Biphosphatase 3); SPHK1 (Sphingosine Kinase 1); NR4A1 (Nuclear Receptor Subfamily 4 Group A Member 1); PLAUR (Plasminogen Activator, Urokinase Receptor); KCNE4 (Potassium Voltage-Gated Channel Subfamily E Regulatory Subunit 4); NFKBIA (NFKB Inhibitor Alpha); TSPAN2 (Tetraspanin 2); RASD2 (RASD Family Member 2); RGCC (Regulator of Cell Cycle); NMB (Neuromedin B)). 

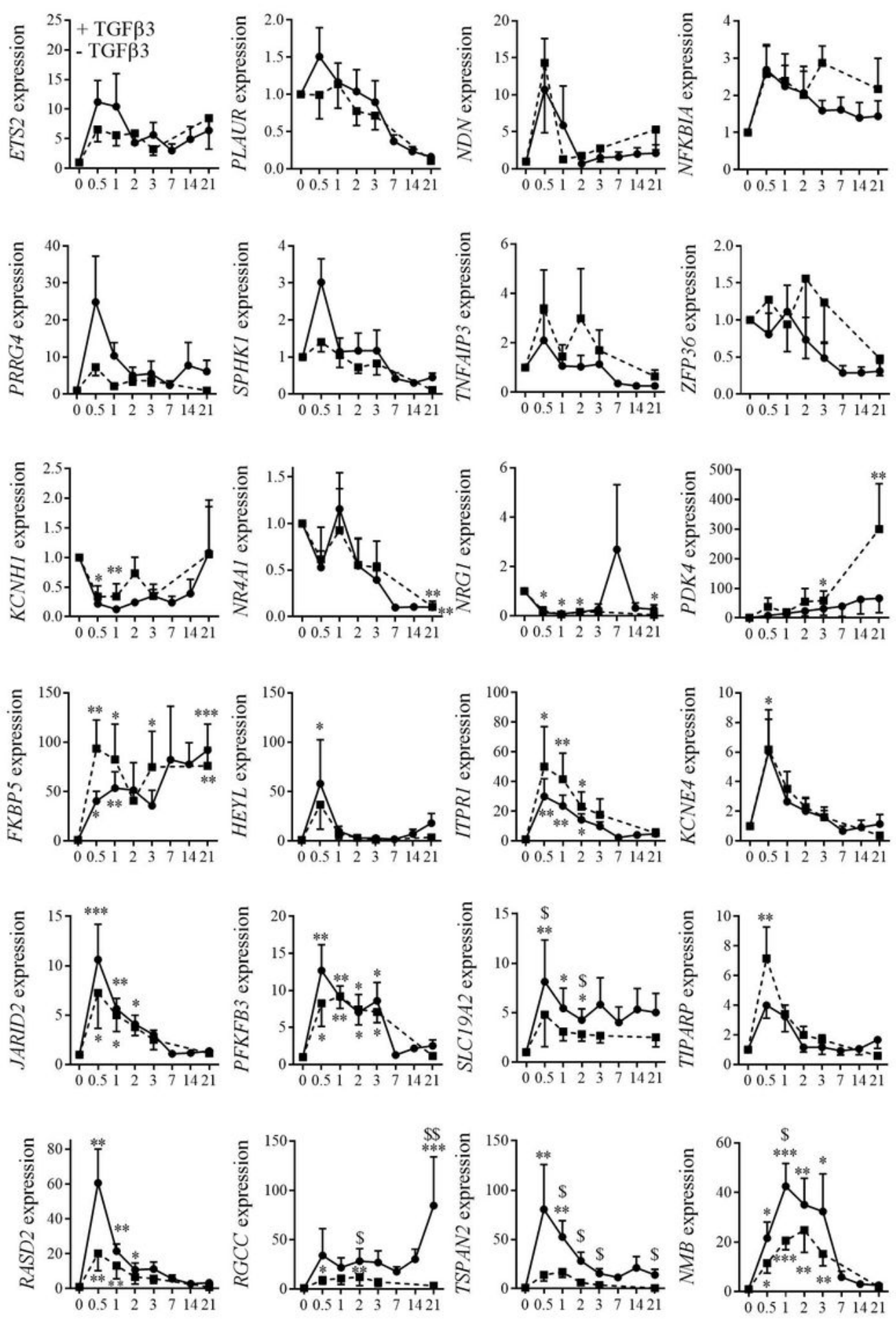

\section{Figure 2}

Expression kinetics of selected genes during chondrogenesis. Expression of selected genes were quantified at different time point in 6 individual BM-MSC replicates during chondrogenesis in pellet conditions with (black line) or without TGF 33 (hatched line) by RT-qPCR. Results are normalized to the expression at day 0 and expressed as the mean \pm sem. Statistical significance of gene expression was indicated as $*: p \leq 0.05, * *: p \leq 0.01, * * *: p \leq 0.001$ when compared to d0 and as $\$: p \leq 0.05, \$ \$: p \leq 0.01$ 
when samples cultured + or - TGF 33 were compared. (HEYL: Hes Related Family BHLH Transcription Factor with YRPW Motif Like; PDK4 (Pyruvate Dehydrogenase Kinase 4); TIPARP (TCDD Inducible Poly(ADP-Ribose) Polymerase); NDN (Necdin, MAGE Family Member); ZFP36 (ZFP36 Ring Finger Protein); TNFAIP3 (TNF Alpha Induced Protein); KCNH1 (Potassium Voltage-Gated Channel Subfamily H Member 1); FKBP5 (FKBP Prolyl Isomerase 5); PRRG4 (Proline Rich And Gla Domain;) ETS2 (ETS ProtoOncogene 2, Transcription Factor); JARID2 (Jumonji And AT-Rich Interaction Domain Containing 2); ITPR1 (Inositol 1,4,5-Trisphosphate Receptor Type 1); SLC19A2 (Solute Carrier Family 19 Member 2); NRG1 (Neuregulin 1); PFKFB3 (6-Phosphofructo-2-Kinase/Fructose-2,6-Biphosphatase 3); SPHK1 (Sphingosine Kinase 1); NR4A1 (Nuclear Receptor Subfamily 4 Group A Member 1); PLAUR (Plasminogen Activator, Urokinase Receptor); KCNE4 (Potassium Voltage-Gated Channel Subfamily E Regulatory Subunit 4); NFKBIA (NFKB Inhibitor Alpha); TSPAN2 (Tetraspanin 2); RASD2 (RASD Family Member 2); RGCC (Regulator of Cell Cycle); NMB (Neuromedin B)).
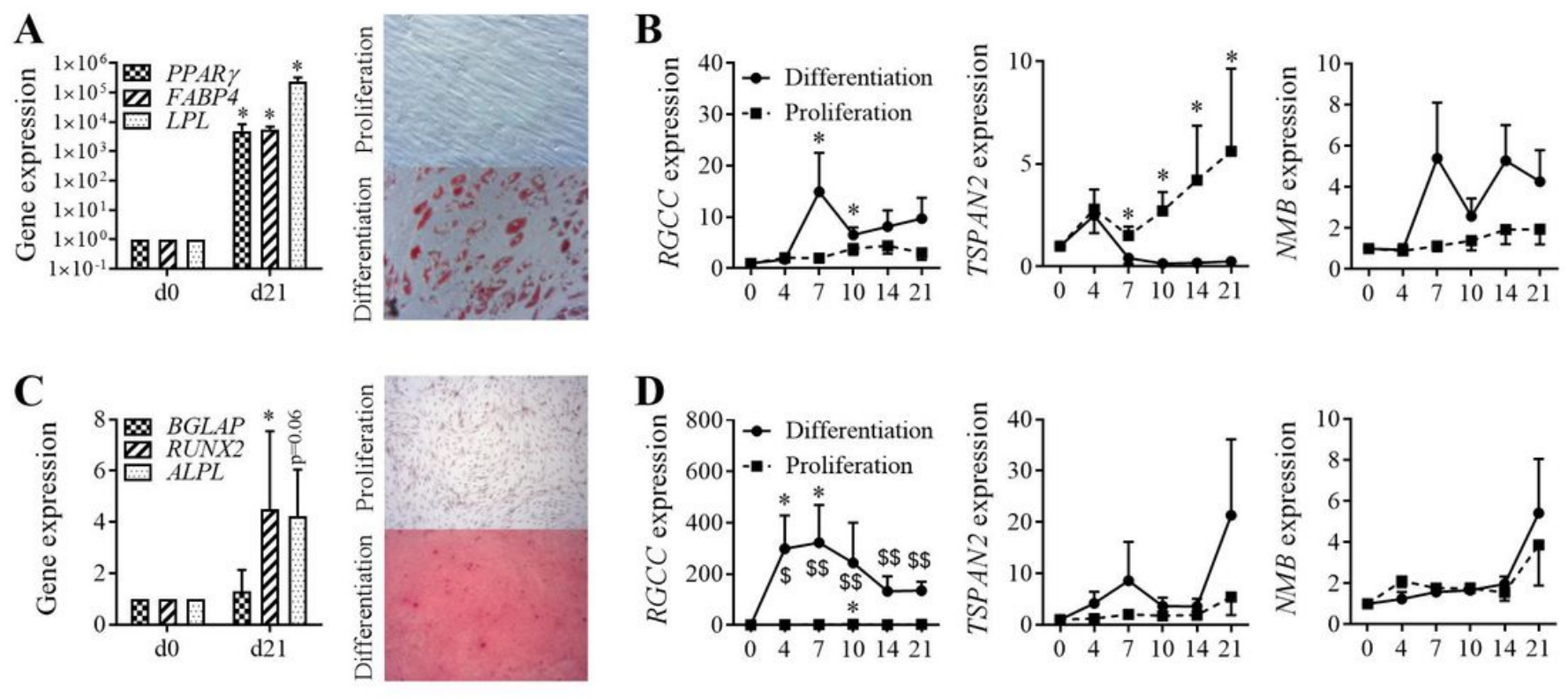

\section{Figure 3}

Relative gene expression during osteogenesis and adipogenesis. a Adipogenic differentiation of BMMSCs characterized by increased expression of peroxysome proliferator-activated receptor (PPAR)- $\gamma$, Fatty Acid Binding Protein (FABP4) and lipoprotein lipase (LPL) and by staining of lipid droplets by Red Oil $O$ in differentiation versus proliferative conditions at day 21. b Relative expression of RGCC, TSPAN2 and NMB at different time points during adipogenic differentiation (black line) or in proliferative conditions (hatched line). c Osteogenic differentiation of BM-MSCs characterized by increased expression of Runx2, osteocalcin (BGLAP), alkaline phosphatase (ALPL) and by Alizarin Red S staining in differentiation versus proliferative conditions at day 21. d Relative expression of RGCC, TSPAN2 and NMB at different time points during osteogenic differentiation (black line) or in proliferative conditions (hatched line). Results are expressed as mean fold change \pm sem and statistical significance was indicated as *: $p \leq 0.05, * *: p \leq 0.01, * \star *: p \leq 0.001$ when samples were compared to day 0 and as $\$$ : 
$p \leq 0.05, \$ \$: p \leq 0.01$ when proliferation and differentiation conditions were compared $(n=6$ biological replicates).
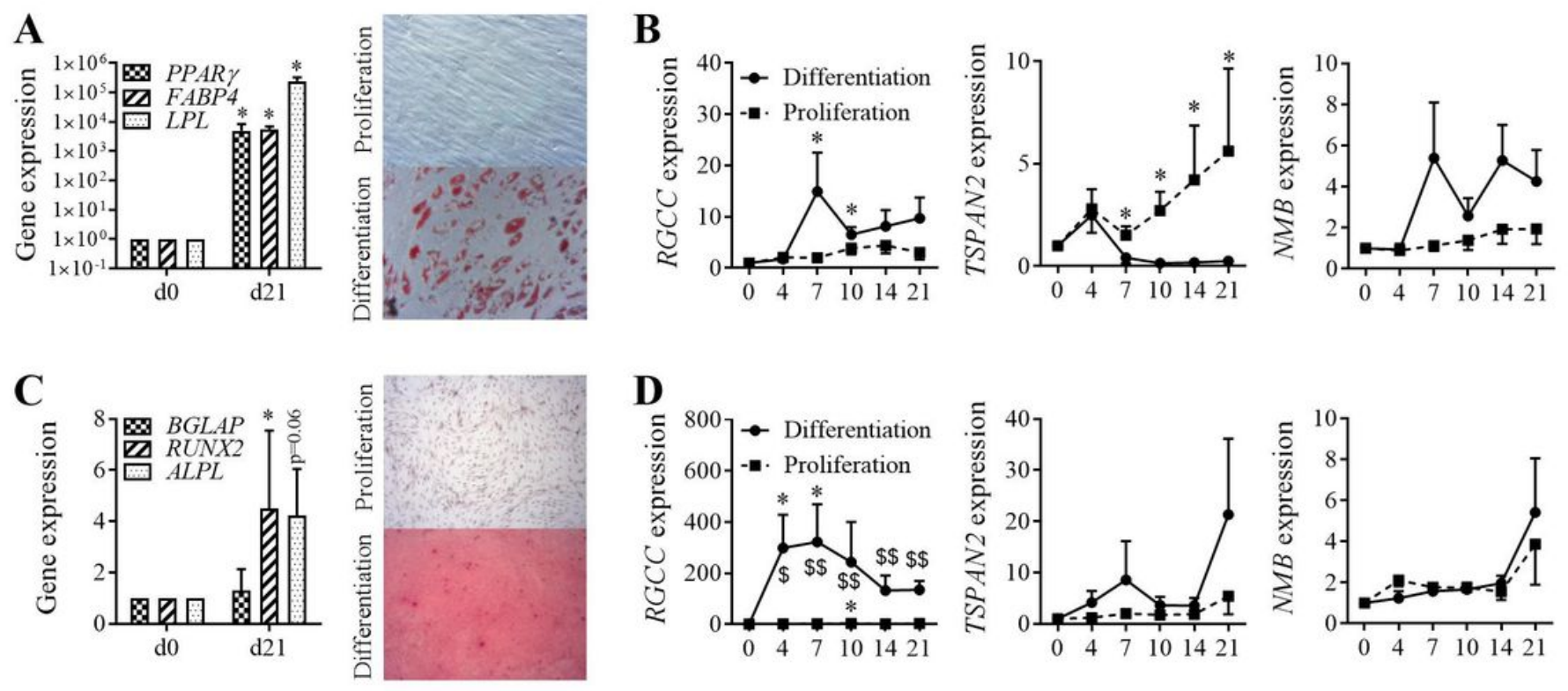

\section{Figure 3}

Relative gene expression during osteogenesis and adipogenesis. a Adipogenic differentiation of BMMSCs characterized by increased expression of peroxysome proliferator-activated receptor (PPAR)- $\gamma$, Fatty Acid Binding Protein (FABP4) and lipoprotein lipase (LPL) and by staining of lipid droplets by Red Oil $O$ in differentiation versus proliferative conditions at day 21. b Relative expression of RGCC, TSPAN2 and NMB at different time points during adipogenic differentiation (black line) or in proliferative conditions (hatched line). c Osteogenic differentiation of BM-MSCs characterized by increased expression of Runx2, osteocalcin (BGLAP), alkaline phosphatase (ALPL) and by Alizarin Red S staining in differentiation versus proliferative conditions at day 21. d Relative expression of RGCC, TSPAN2 and NMB at different time points during osteogenic differentiation (black line) or in proliferative conditions (hatched line). Results are expressed as mean fold change \pm sem and statistical significance was indicated as *: $p \leq 0.05, * *: p \leq 0.01, * * *: p \leq 0.001$ when samples were compared to day 0 and as $\$$ : $p \leq 0.05$, $\$$ : $p \leq 0.01$ when proliferation and differentiation conditions were compared $(n=6$ biological replicates). 
A

NMB

Actin

d0 $\mathrm{d} 3$

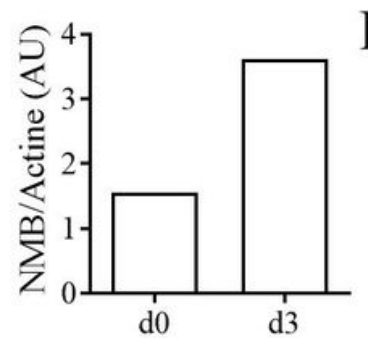

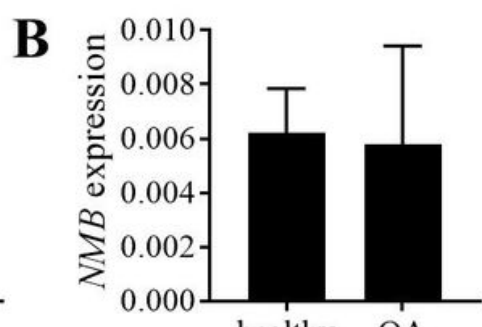

healthy $\mathrm{OA}$

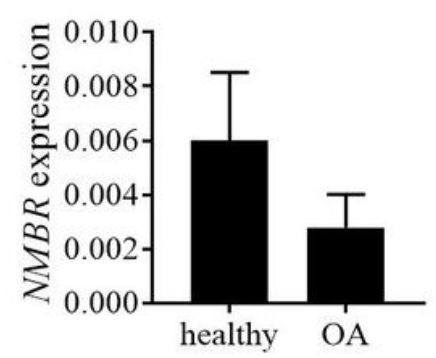

C
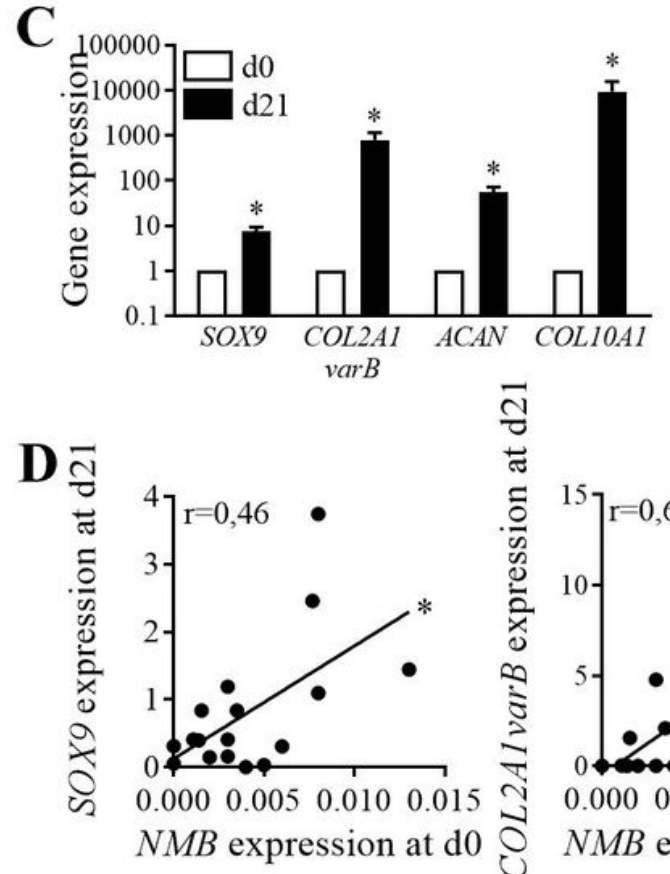
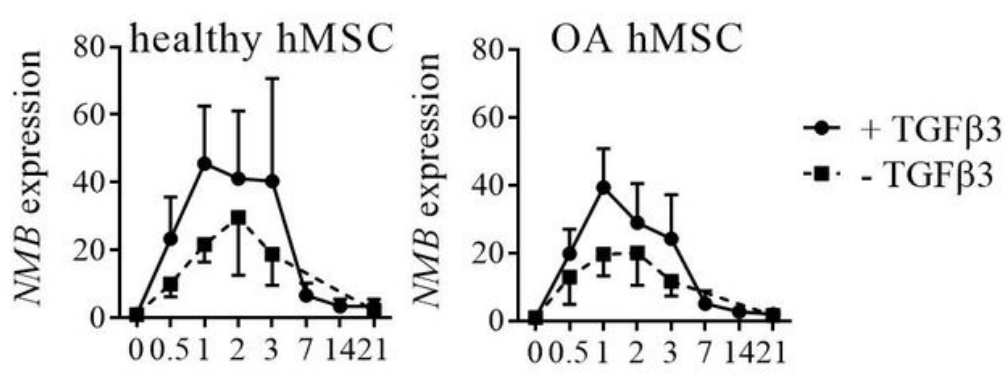
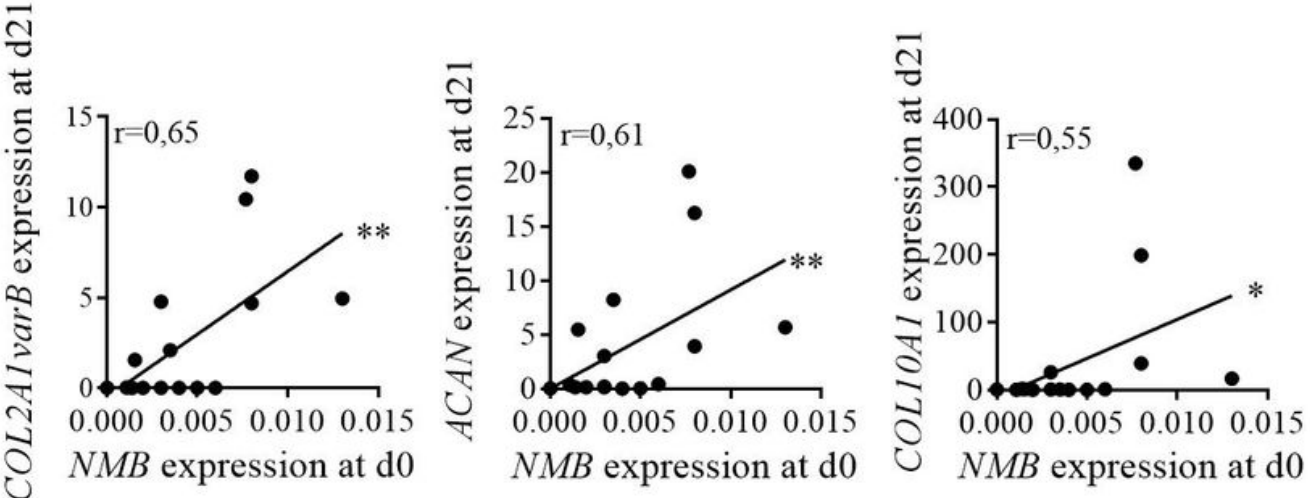

Figure 4

NMB expression during chondrogenesis. a Detection of NMB (13 KDa) and $\beta$-actin (45 KDa) proteins in BM-MSCs at $\mathrm{d} 0$ and micropellets at $\mathrm{d} 3$ by Western blotting (left panel) and their respective quantification (right panel). b Relative expression of NMB and NMBR in BM-MSCs from healthy and OA donors quantified by RT-qPCR. Results are expressed as mean \pm sem ( $n=3$ biological replicates). $c$ Chondrogenic differentiation of BM-MSCs characterized by increased gene expression of SOX9, collagen type IIB (COL2A1varB), aggrecan (ACAN) and collagen type X (COL10A1) at d21 compared to d0 (left panel) ( $n=6$ biological replicates). Kinetics of NMB expression during chondrogenesis of BM-MSCs isolated from healthy (middle panel) or OA (right panel) donors and cultured with or without TGF $\beta 3$ ( $n=3$ biological replicates by group of donors). $\mathrm{d}$ Correlation between NMB expression in BM-MSCs at $\mathrm{d} 0$ and chondrogenic markers (SOX9, COL2A1varB, ACAN, COL10A1) at d21 of chondrogenic differentiation $(n=17) . *: p \leq 0.05, * *: p \leq 0.01$. 
A

NMB

Actin

d0 $\mathrm{d} 3$

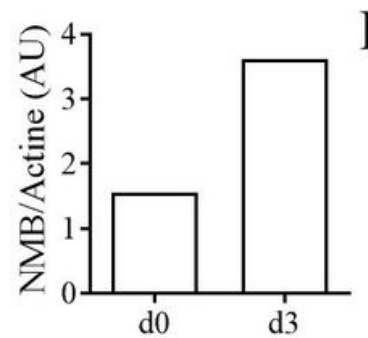

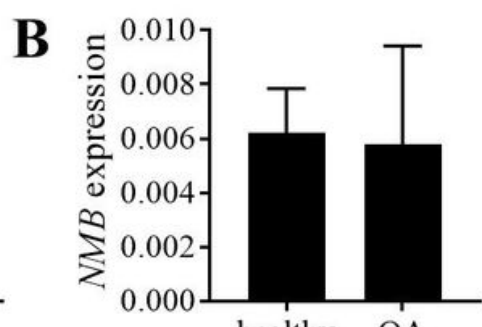

healthy $\mathrm{OA}$

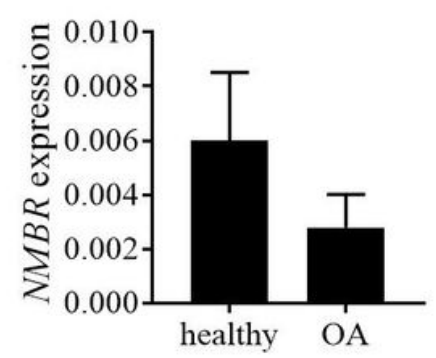

C
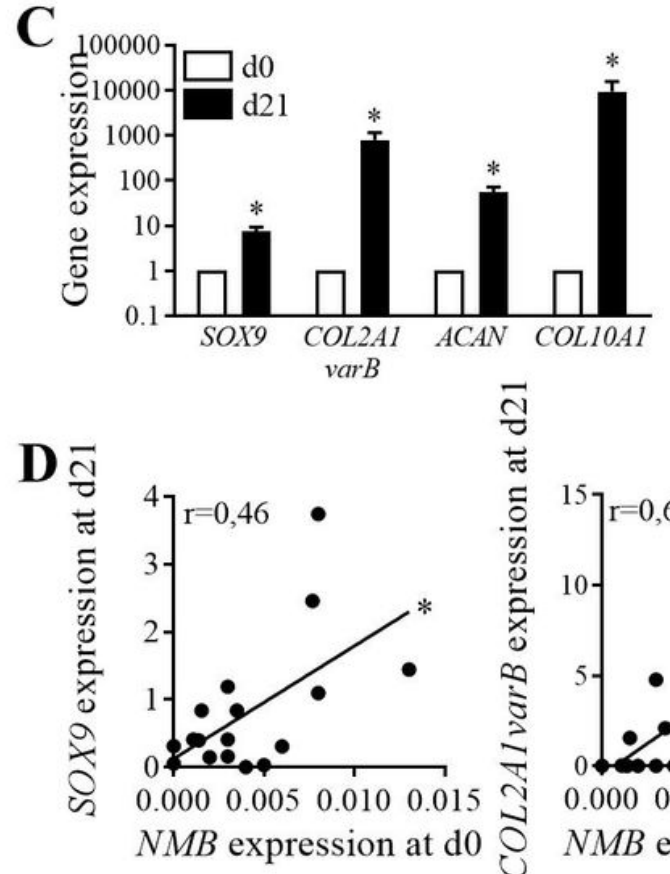
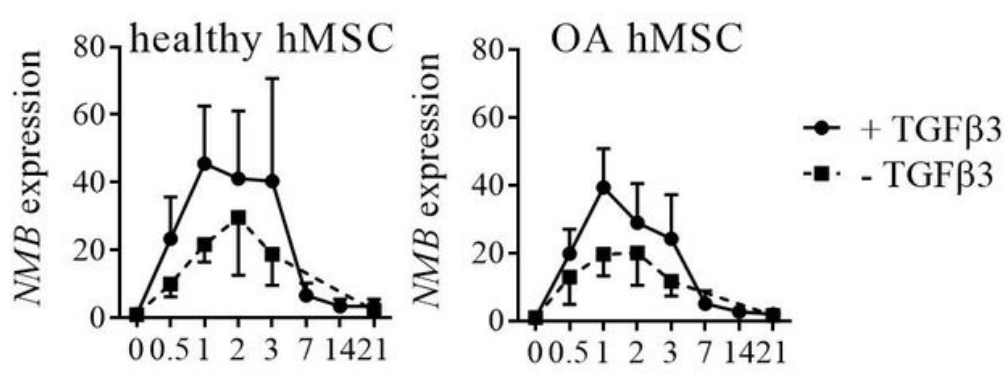
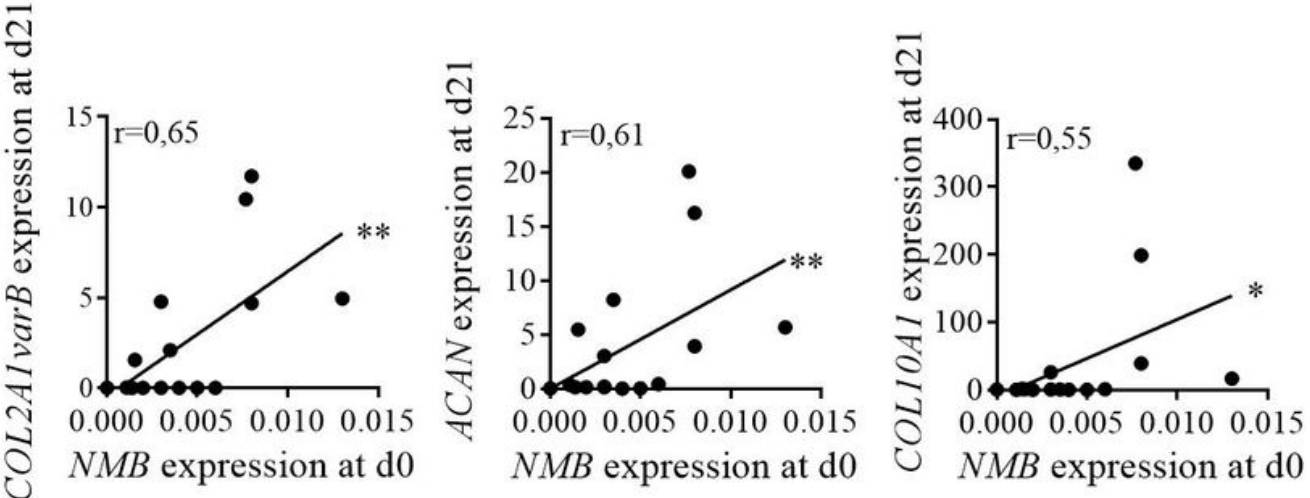

Figure 4

NMB expression during chondrogenesis. a Detection of NMB (13 KDa) and $\beta$-actin (45 KDa) proteins in BM-MSCs at $\mathrm{d} 0$ and micropellets at $\mathrm{d} 3$ by Western blotting (left panel) and their respective quantification (right panel). b Relative expression of NMB and NMBR in BM-MSCs from healthy and OA donors quantified by RT-qPCR. Results are expressed as mean \pm sem ( $n=3$ biological replicates). $c$ Chondrogenic differentiation of BM-MSCs characterized by increased gene expression of SOX9, collagen type IIB (COL2A1varB), aggrecan (ACAN) and collagen type X (COL10A1) at d21 compared to d0 (left panel) ( $n=6$ biological replicates). Kinetics of NMB expression during chondrogenesis of BM-MSCs isolated from healthy (middle panel) or OA (right panel) donors and cultured with or without TGF $\beta 3$ ( $n=3$ biological replicates by group of donors). $\mathrm{d}$ Correlation between NMB expression in BM-MSCs at $\mathrm{d} 0$ and chondrogenic markers (SOX9, COL2A1varB, ACAN, COL10A1) at d21 of chondrogenic differentiation $(n=17) . *: p \leq 0.05, * *: p \leq 0.01$. 

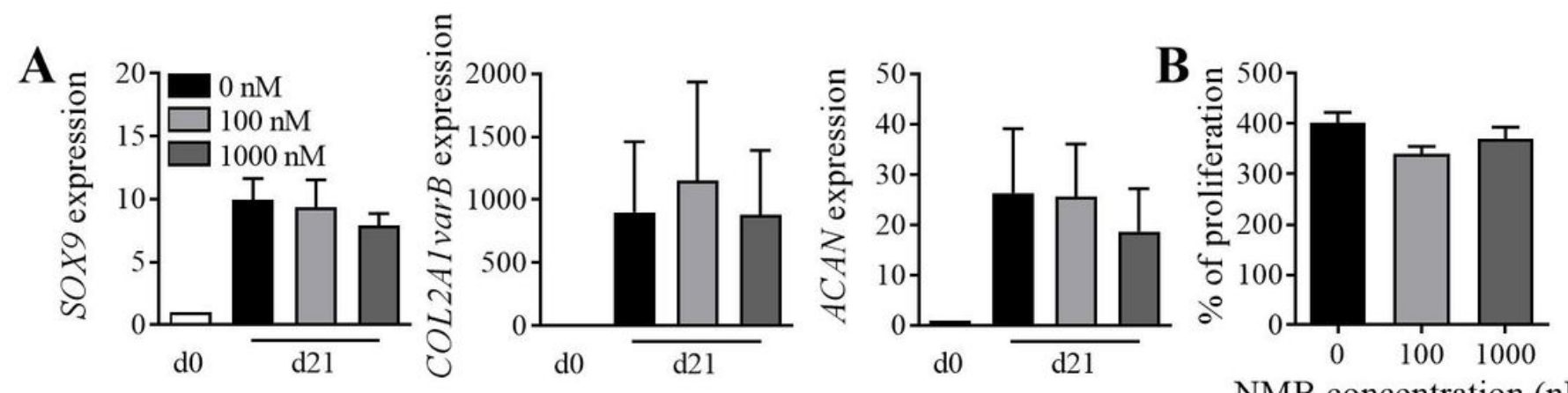

NMB concentration $(\mathrm{nM})$
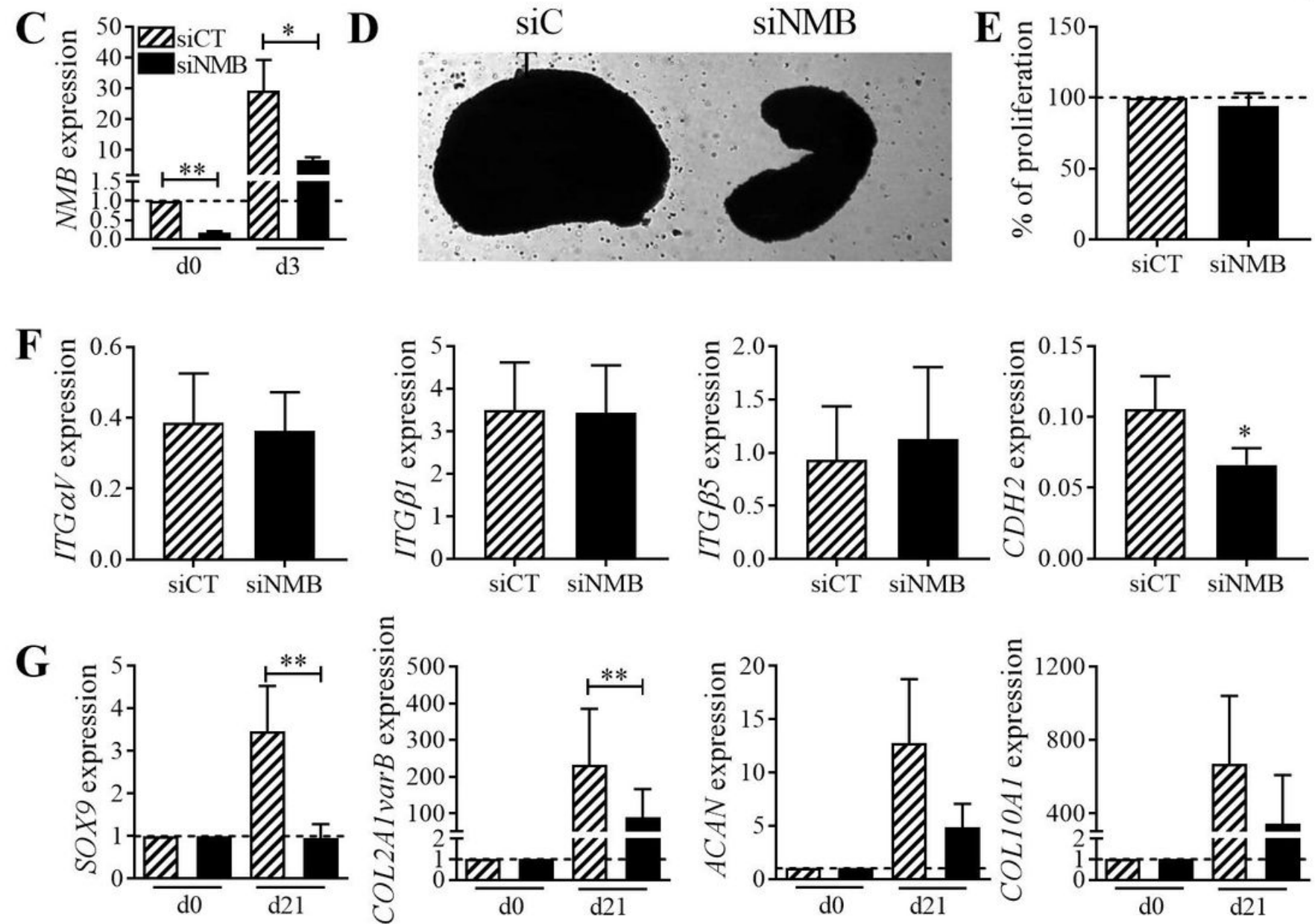

Figure 5

Role of NMB during chondrogenesis. a Fold change expression of chondrocyte markers after 21 days of chondrogenic differentiation of BM-MSCs with different concentrations of recombinant NMB. Results are expressed as mean fold change \pm SEM ( $n=3$ biological replicates). b Proliferation of BM-MSCs cultured with different concentrations of recombinant NMB after 5 days of culture. Results are expressed as the mean percentage \pm sem ( $n=4$ biological replicates). $c$ Fold change expression of NMB in BM-MSCs at day 3 after transfection with siRNA control (siCT) or siRNA against NMB (siNMB). (normalized to NMB expression at d0). $d$ Representative picture of micropellets after 3 days of chondrogenic differentiation of 
BM-MSCs transfected with siCT or siNMB. e Proliferation of BM-MSCs transfected with siCT or siNMB after 3 days of culture in monolayer. Results are expressed as mean percentage \pm sem $(n=4$ biological replicates). $f$ Relative expression of adhesion molecules in BM-MSCs transfected with siCT or siNMB after 3 days of chondrogenic differentiation. g Fold change expression of chondrocyte markers in BMMSCs transfected with siCT or siNMB at day 21 of chondrogenesis. Results are expressed as mean \pm sem ( $n=7$ biological replicates). *: $p \leq 0.05,{ }^{*}: p \leq 0.01$.
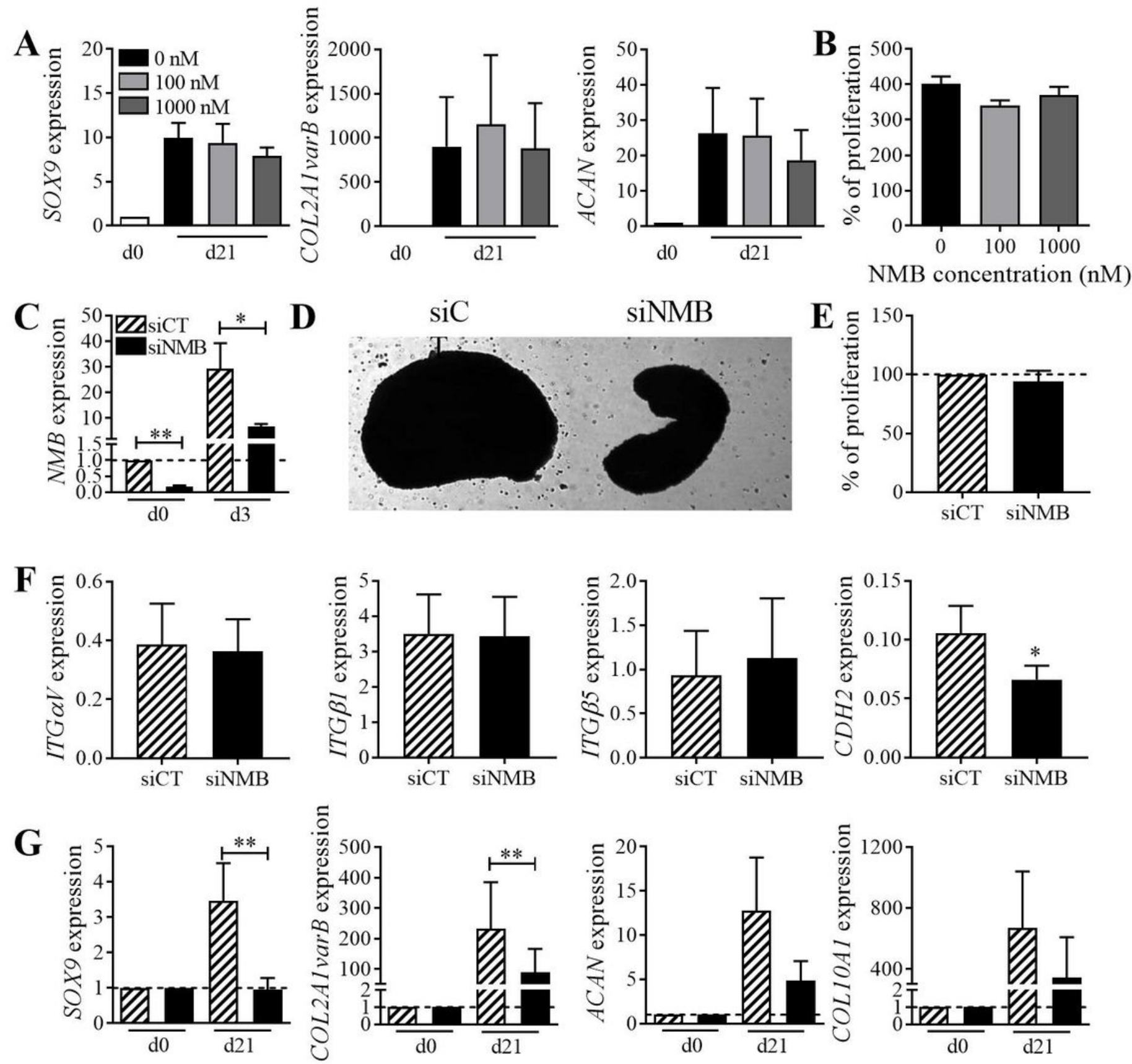

Figure 5 
Role of NMB during chondrogenesis. a Fold change expression of chondrocyte markers after 21 days of chondrogenic differentiation of BM-MSCs with different concentrations of recombinant NMB. Results are expressed as mean fold change \pm SEM ( $n=3$ biological replicates). b Proliferation of BM-MSCs cultured with different concentrations of recombinant NMB after 5 days of culture. Results are expressed as the mean percentage \pm sem ( $n=4$ biological replicates). c Fold change expression of NMB in BM-MSCs at day 3 after transfection with siRNA control (siCT) or siRNA against NMB (siNMB). (normalized to NMB expression at $d 0$ ). $d$ Representative picture of micropellets after 3 days of chondrogenic differentiation of BM-MSCs transfected with siCT or siNMB. e Proliferation of BM-MSCs transfected with siCT or siNMB after 3 days of culture in monolayer. Results are expressed as mean percentage \pm sem ( $n=4$ biological replicates). $f$ Relative expression of adhesion molecules in BM-MSCs transfected with siCT or siNMB after 3 days of chondrogenic differentiation. g Fold change expression of chondrocyte markers in BMMSCs transfected with siCT or siNMB at day 21 of chondrogenesis. Results are expressed as mean \pm sem ( $n=7$ biological replicates). *: $p \leq 0.05, * *: p \leq 0.01$.
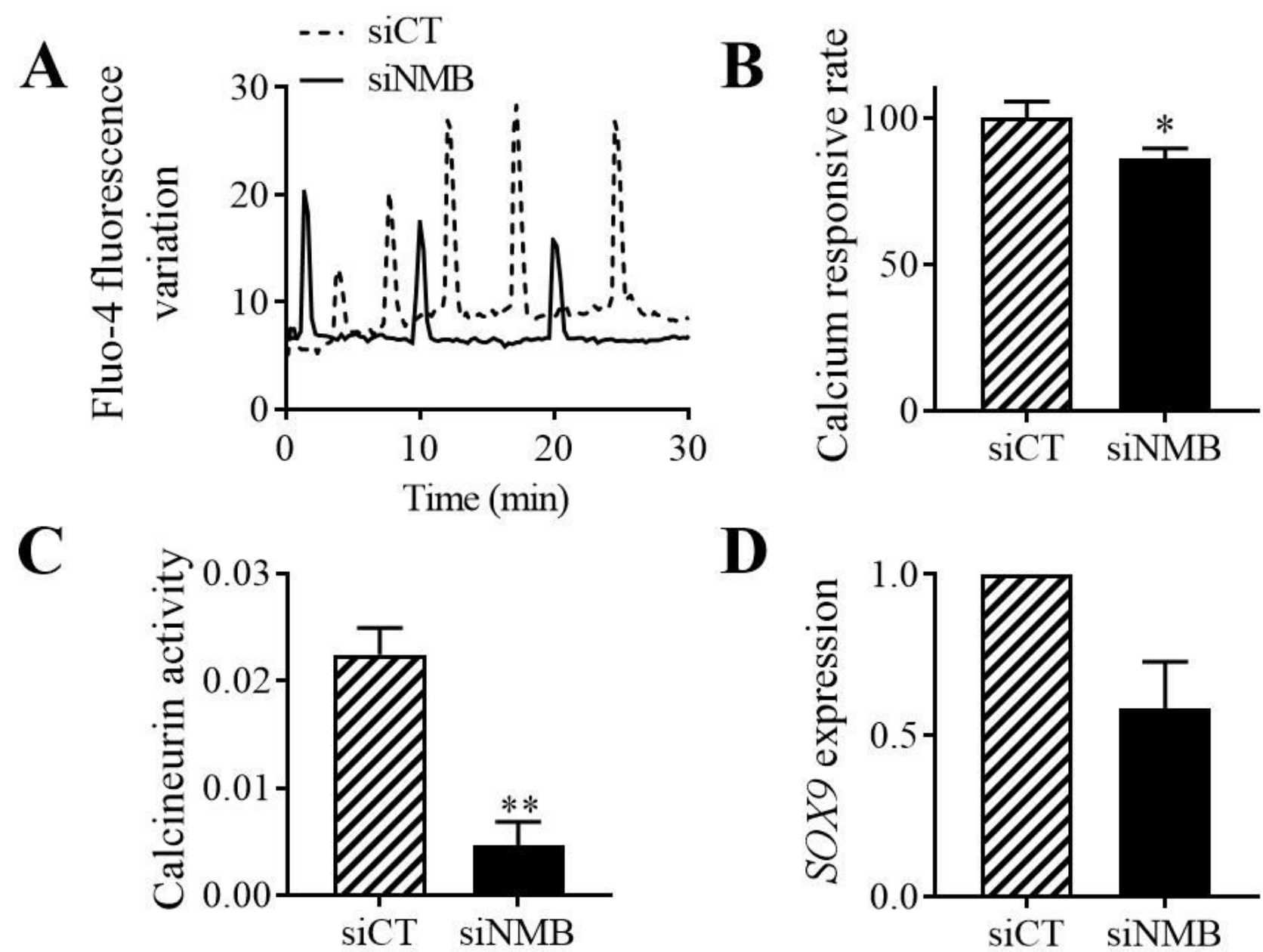

D

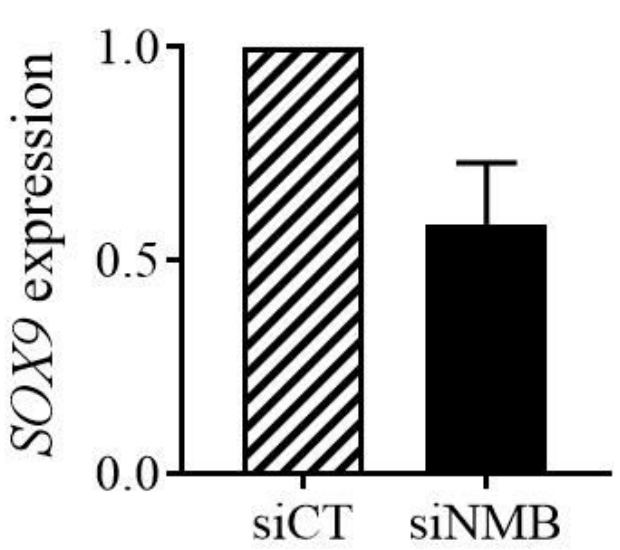

Figure 6 
Role of NMB in calcium signaling. a Time course of maximal calcium intensity of spontaneous calcium oscillations in BM-MSCs transfected with siCT or siNMB and loaded with Fluo-4-AM at day 3 of chondrogenesis. b Calcium responsive rate determined in BM-MSCs transfected with siCT or siNMB at day 3 of chondrogenesis and expressed as fold change rate. $c$ Intracellular calcineurin phosphatase activity determined in BM-MSCs transfected with siCT or siNMB at day 3 of chondrogenesis. $d$ Fold change expression of SOX9 in BM-MSCs transfected with siCT or siNMB at day 3 of chondrogenesis. *: $p$ $\leq 0.05, * *: p \leq 0.01$.
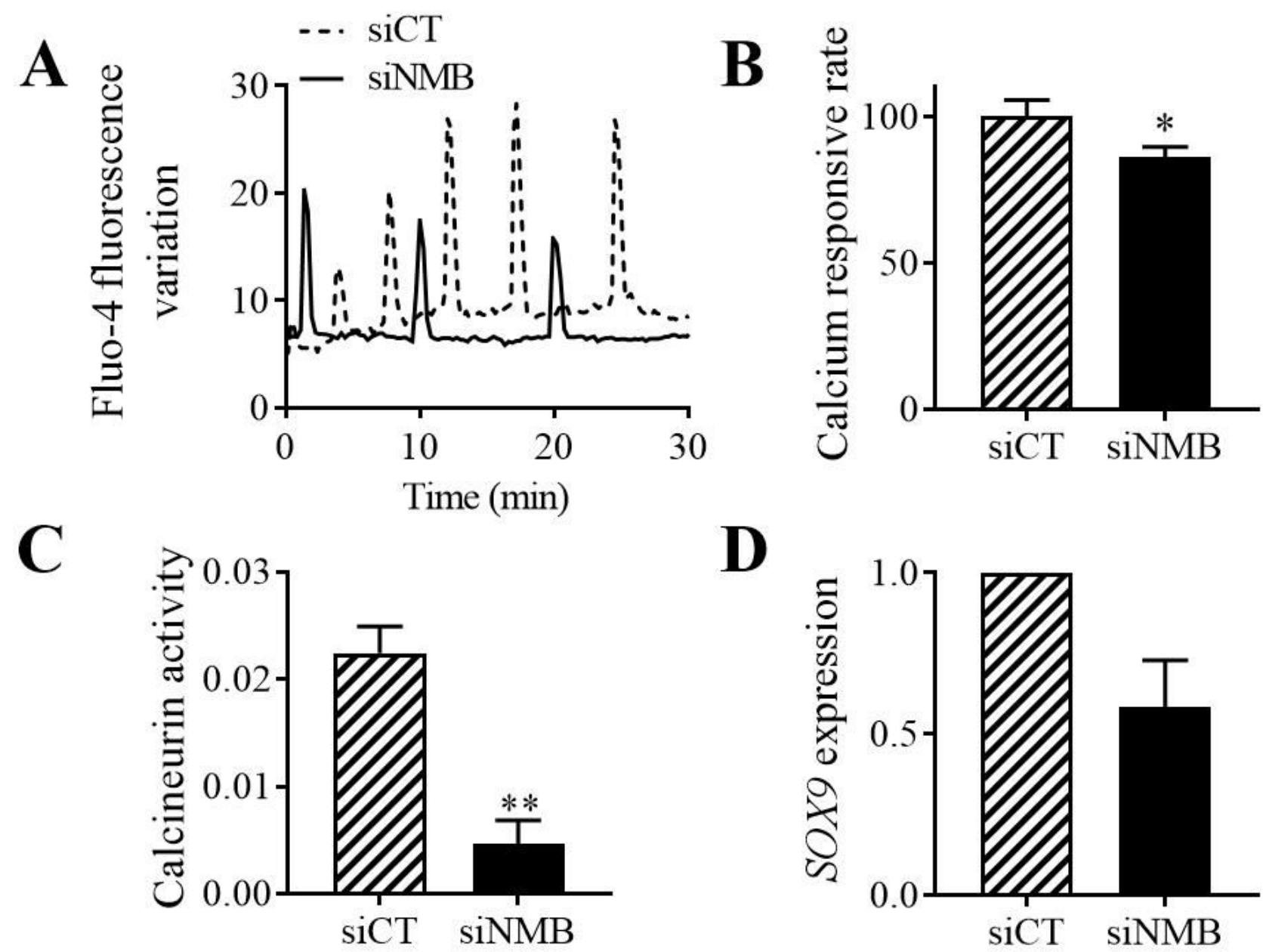

D

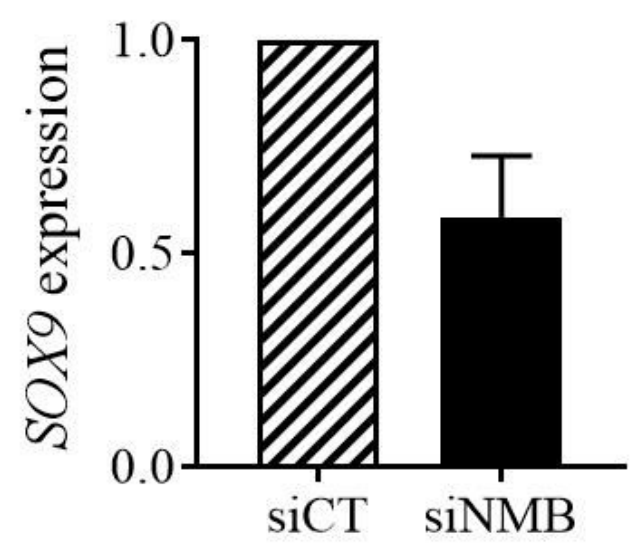

Figure 6

Role of NMB in calcium signaling. a Time course of maximal calcium intensity of spontaneous calcium oscillations in BM-MSCs transfected with siCT or siNMB and loaded with Fluo-4-AM at day 3 of chondrogenesis. b Calcium responsive rate determined in BM-MSCs transfected with siCT or siNMB at day 3 of chondrogenesis and expressed as fold change rate. $c$ Intracellular calcineurin phosphatase activity determined in BM-MSCs transfected with siCT or siNMB at day 3 of chondrogenesis. $d$ Fold 
change expression of SOX9 in BM-MSCs transfected with siCT or siNMB at day 3 of chondrogenesis. *: $p$ $\leq 0.05, * *: p \leq 0.01$.

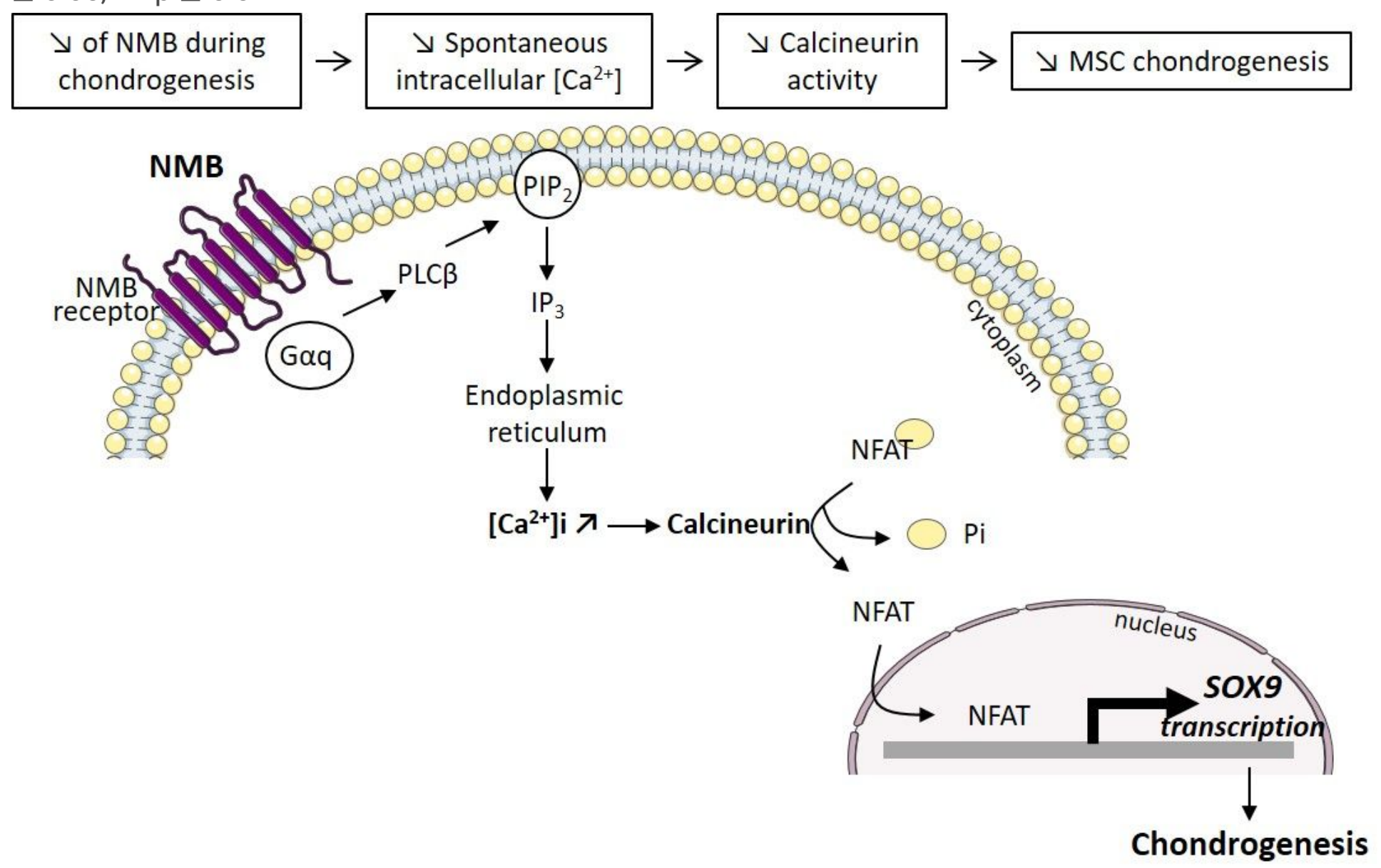

\section{Figure 7}

Suggested signal transduction pathway of NMB in chondroprogenitor cells. Upon binding of NMB to its receptor, phospholipase (LPC) is activated and catalyzes the hydrolysis of phosphatidyl inositol 4,5bisphosphate (PIP2) into inositol 1,4,5-triphosphate (IP3). Binding of IP3 to the receptors in the endoplasmic reticulum triggers $\mathrm{Ca} 2+$ release in the cytosol. Intracellular $\mathrm{Ca} 2+$ influx activates calcineurin, which dephosphorylates nuclear factor of the activated T-cell (NFAT) allowing its nuclear translocation and binding to the SOX9 promoter. 

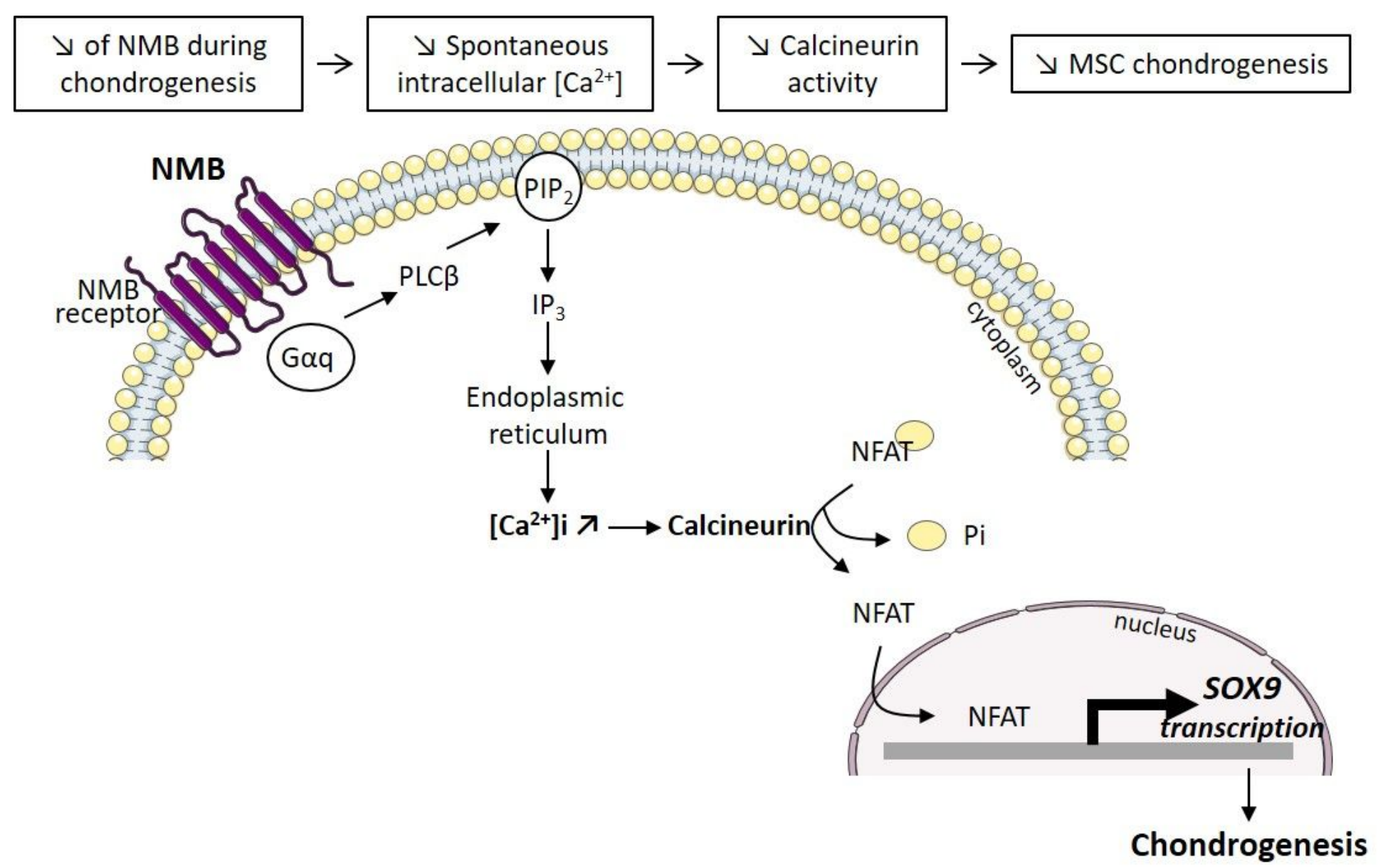

Figure 7

Suggested signal transduction pathway of NMB in chondroprogenitor cells. Upon binding of NMB to its receptor, phospholipase (LPC) is activated and catalyzes the hydrolysis of phosphatidyl inositol 4,5bisphosphate (PIP2) into inositol 1,4,5-triphosphate (IP3). Binding of IP3 to the receptors in the endoplasmic reticulum triggers $\mathrm{Ca} 2+$ release in the cytosol. Intracellular $\mathrm{Ca} 2+$ influx activates calcineurin, which dephosphorylates nuclear factor of the activated T-cell (NFAT) allowing its nuclear translocation and binding to the SOX9 promoter.

\section{Supplementary Files}

This is a list of supplementary files associated with this preprint. Click to download.

- Additionalfile1.jpg

- Additionalfile1.jpg 